\title{
Numerical investigation of viscous effects on the gap resonance between side-by-side barges
}

\author{
X. Feng ${ }^{\mathrm{a}, \mathrm{c}}$, W. Bai ${ }^{\mathrm{b}, *}$, X.B. Chen ${ }^{\mathrm{c}}$, L. Qian ${ }^{\mathrm{b}}, \mathrm{Z} \cdot \mathrm{Ma}^{\mathrm{b}}$ \\ ${ }^{a}$ Department of Civil and Environmental Engineering, National University of Singapore, Kent Ridge, Singapore 117576, \\ Singapore \\ ${ }^{b}$ School of Computing, Mathematics and Digital Technology, Manchester Metropolitan University, Chester Street, \\ Manchester M1 5GD, UK \\ ${ }^{c}$ Bureau Veritas Singapore, 20 Science Park Road, Science Park II, Singapore
}

\begin{abstract}
This paper presents a numerical study of the gap resonance between two side-by-side barges by using a multiphase Navier-Stokes equations model. In order to verify the multiphase flow model, it is firstly applied to simulate a two-dimensional gap resonance problem for two fixed boxes under various wave conditions. A comparison of the free surface elevations obtained on successively refined grids confirms the mesh convergence of numerical solutions. The calculated wave elevation response amplitude operators (RAOs) in the gap compare well with the experimental measurements. The multiphase flow model is further extended to calculate a three-dimensional gap resonance problem for two adjacent rectangular barges. The computed free surface RAOs in the gap also agree well with the experimental results. A close examination of the flow velocity and vorticity in the gap region at the piston resonant mode reveals that large amount of vortices are generated by the sharp corners of the two barges and shed downwards, which provide an effective mechanism to dissipate the flow kinematic energy and to reduce the wave elevation in the gap. On the contrary, rounded corners are not able to induce the same level amount of vortices to dampen the gap resonance. The effects of incident wave steepness on the viscous damping associated with the twin-barge system are highlighted.
\end{abstract}

Keywords: Computational Fluid Dynamics, Side-by-side, Gap resonance, Viscous damping, Wave tank

\section{Introduction}

As the oil and gas industry is moving towards remote locations and deeper waters, Floating Production Storage and Offloading (FPSO) and Floating Liquefied Natural Gas (FLNG) systems have become economically attractive for offshore field development. Side-by-side offloading from FPSO or FLNG to a shuttle carrier dramatically reduces the cost of building a long pipeline for locations far from any coastal terminal. Technical challenges, however, arise for such multibody floating systems, due to the complexity of multibody hydrodynamics. One of the critical issues might be the wave resonances in the narrow gap between two

\footnotetext{
* Corresponding author

Email address: w.bai@mmu.ac.uk (W. Bai)
} 
side-by-side vessels. The gap resonances occur at some particular wave frequencies and are characterized by remarkably amplified wave motions and body responses.

Potential flow models are predominantly used in the industry to study this problem of wave interaction with multiple floating bodies. Theoretical solutions of the resonant modes of fluids trapped between simple bodies were derived by Molin (2001) and Yeung and Seah (2007), in the framework of linear potential flow model. It was reported in Molin (2001) that both the piston and longitudinal sloshing modes of gap resonance can be excited. In the piston mode resonance, the fluid in the gap is pumped up and down as a 'rigid' body with only one peak response along the gap; while the sloshing modes show a near sinusoidal free surface along the gap with several crests and troughs. The pumping or piston mode is found more critical than the longitudinal sloshing modes, in the perspective of fluid motion amplification. The gap resonance discussed in this study refers to the piston mode unless otherwise specified. Linear as well as second-order models have been developed to numerically simulate two adjacent barges or vessels, including Newman and Sclavounos (1988), Kashiwagi et al. (2005), Teigen and Niedzwecki (2006), Lewandowski (2008), Zhu et al. (2008) and Sun et al. (2010). While gap resonant frequencies can be accurately predicted, linear potential models tend to over-estimate the wave responses at the piston mode, according to comparisons with experimental tests.

In attempt to address the discrepancy between the model tests and linear predictions, Feng and Bai (2015), Li and Zhang (2016) developed time-domain fully nonlinear potential flow models to investigate the diffraction and radiation problems for gap resonance respectively. In Feng and Bai (2015), it was reported that the free surface nonlinearity does not account for the over-estimation of resonant responses. An experimental study was conducted by Zhao et al. (2017) who excited first and higher harmonic components of the resonant response in NewWaves. The unrealistic prediction is related to the small damping near the resonant frequency in potential flow models. Several approaches have been developed to suppress this unrealistic prediction. Huijsmans et al. (2001) and Buchner et al. (2001) put a rigid lid on the free surface between side-by-side moored vessels. This 'lid method' effectively suppresses the amplified gap free surface, yet holds no physical meaning. Newman (2004) modelled a movable damping lid on the gap free surface and used a generalized modal technique to compute the lid motions. Instead of performing only numerical treatments, Chen (2005) modified the free surface boundary conditions by introducing a damping 'force' term. The damping term acts analogously as energy dissipation that is not taken into account in potential flow models. This method was proven practically effective by Fournier et al. (2006) and Pauw et al. (2007). However, one has to calibrate the unknown dissipation coefficient using measurement data, and there is no a priori theory to determine it. More recently, Watai et al. (2015) and Ganesan and Sen (2016) employed Chen's method in their time-domain model for two side-by-side vessels and again the damping coefficient was calibrated from tests. While predictions of resonant surface elevation in the gap can be improved through calibration, the physical reason of the discrepancy between predictions and experiments might not be addressed in the framework of potential flow.

Viscous flow models based on the Navier-Stokes equations have the capabilities of capturing vortex 
shedding, flow separation and energy dissipation in the bulk of fluid domain, and could provide better understandings of the damping mechanism associated with the piston gap resonance. For prediction of the resonant response, Lu et al. (2010) demonstrated that viscous models provide much improved results than potential flow models in a 2D case of two side-by-side boxes. Similar cases were studied in Moradi et al. (2016) using a NS solver with a focus on the effect of water depth on dynamics of gap resonance. Based on the understanding of energy dissipation in the $2 \mathrm{D}$ gap through viscous models, an energy dissipation model was proposed recently in Chua et al. (2016) through the analysis of energy conservation in a control volume of fluid around the bodies. By comparison between the linear potential flow model with the viscous model, a proper dissipation coefficient in Chen's model can be determined. A link between the energy loss coefficient in the energy dissipation model and the dissipation coefficient in Chen's model was then constructed in Tan et al. (2017). This shows a possible method to determine the dissipation coefficient without the calibration but through an iterative process. To quantify the viscous dissipation upon resonance, Faltinsen and Timokha (2015) presented a pressure discharge model resulting in a modified dynamic free surface condition. By applying a pressure drop coefficient, they achieved good agreements with tests without any priori. Nonetheless, most of the studies are mainly in the 2D framework. Meanwhile, Kristiansen and Faltinsen (2008) employed a vortex-tracking method to study a 2D moonpool formed by two rectangular hulls undergoing heave motions. The vortex tracking analysis revealed that flow separation around the sharp body corners/edges at the gap entrance is the main reason for the discrepancy between potential models and measurements. Their later investigation using a domain-decomposition model in Kristiansen and Faltinsen (2012) confirmed this conclusion. It is worthwhile to mention that the domain-decomposition model combines a potential flow solver in the outer domain and a viscous flow solver in the inner domain. It was demonstrated again that the inner viscous flow model is effective in capturing the viscous damping effect around the floating bodies.

While some understanding of the discrepancy has been created in simple 2D cases of boxes from previous studies, no much light has been thrown on the similar gap resonance associated with a 3D vessels/barges system. It is understood in Feng and Bai (2015) that the discrepancy of peak response between the potential flow model and experimental data in a 3D twin-barge system may not be as high as that in a 2D twinbox system. In this sense, detailed investigations on flow structures (including flow separation and vortex shedding) in the gap resonant fluid around two floating vessels are essential, which will be more helpful for better understanding the damping mechanism in a more realistic situation. More importantly, no work has been published to discuss the effect of incident wave amplitude on the gap resonant responses. This is because RAOs (Response Amplitude Operators) generated from the linear potential flow model are independent of the incident wave amplitude. This independence is mostly true for wave frequencies away from the gap resonance. However, the incident wave amplitude clearly affects the strength of system damping at the gap resonance. Further study on the effect of incident wave amplitude on the gap resonance is needed.

This work attempts to investigate the flow structures around two 3D side-by-side barges upon gap res- 
onances and to shed light on the damping mechanism at the piston mode. The effect of incident wave amplitude on the resonant response is highlighted for barges with both square and rounded bilges. To capture the local flow characteristics near the gap, a viscous flow solver is essential. We build the numerical model based on an open source CFD package OpenFOAM ${ }^{\circledR}$ and implement the model on the massive parallel computing system in the National Supercomputing Centre Singapore.

This paper is organized as follows. Section 2 briefly presents the governing equations of a viscous numerical wave tank, the boundary conditions and the techniques for free surface capturing. Numerical implementation of wave generation and absorption is also described. Section 3 validates the numerical model via a simple $2 \mathrm{D}$ case of two boxes in a wave flume. Section 4 simulates two 3D side-by-side barges floating in a numerical wave basin. Flow characteristics near the barge bilges at the piston mode gap resonance are demonstrated. Comparisons are made between cases of square and rounded bilges. Effect of incident wave amplitude is further investigated by varying wave steepness. Concluding remarks are drawn in Section 5

\section{Numerical model}

The viscous flow model solves the incompressible Navier-Stokes (N-S) equations for a two-phase flow of water and air. A volume of fluid (VOF) technique is incorporated for capturing free surface. The N-S equations are solved by a finite volume method (FVM). The wave tank model is based on the OpenFOAM ${ }^{\circledR}$ multiphase solver 'interFoam'. A wave generation utility 'waves2Foam' presented in Jacobsen et al. (2012) is incorporated to generate propagating incident waves and to dissipate far field reflection waves.

\subsection{Governing equations}

The continuity equation for incompressible flows reads:

$$
\frac{\partial \rho}{\partial t}+\nabla \cdot(\rho \mathbf{u})=0
$$

and the Navier-Stokes equations are written as

$$
\frac{\partial \rho \mathbf{u}}{\partial t}+\nabla \cdot\left(\rho \mathbf{u u}^{T}\right)=-\nabla P-(\mathbf{g} \cdot \mathbf{x}) \nabla \rho+\nabla \cdot(\mu \nabla \mathbf{u})+\sigma_{t} k_{\alpha} \nabla \alpha,
$$

where $\mathbf{u}=(u, v, w)$ is the velocity field in Cartesian coordinates, $\rho$ the fluid density, $P$ the pressure in excess of the hydrostatic part and $\mathbf{g}$ the gravitational acceleration. $\mu$ is the dynamic viscosity of the fluid, $\sigma_{t}$ the surface tension coefficient and $k_{\alpha}$ the surface curvature, see Ubbink and Issa (1999). The surface tension coefficient between air and water at 20 degree is $0.074 \mathrm{~kg} / \mathrm{s}^{2}$. Note that $\rho=\rho(\mathbf{x})$ varies with the volume fraction of water $\alpha$ in the computational cells. The above equations are solved for the two-phase flow simultaneously, and the mixed fluids are tracked using the scalar field $\alpha$ which is 0 for air and 1 for water. Any intermediate value between 0 and 1 represents a mixture of the two fluids. In the VOF method, the distribution of phase fraction indicator $\alpha$ is modelled by the following transport equation for the volume fraction of water,

$$
\frac{\partial \alpha}{\partial t}+\nabla \cdot[\alpha \mathbf{u}]=0
$$


However, accurate calculation of the phase fraction distribution is a critical issue in numerical simulations. The interface region between the two phases tends to be smeared over a few grid cells and is highly sensitive to mesh resolution. To assure boundedness and conservativeness of the phase volume fraction, an additional convective term is introduced into Eq. (3),

$$
\frac{\partial \alpha}{\partial t}+\nabla \cdot[\alpha \mathbf{u}]+\nabla \cdot\left[\alpha(1-\alpha) \mathbf{u}_{r}\right]=0
$$

where $\mathbf{u}_{r}=\mathbf{u}_{\text {water }}-\mathbf{u}_{\text {air }}$ is a relative velocity between the water and air and termed as the 'compression velocity'. The last term on the left hand side of the above equation is often referred to as the interface compression term, which limits the smearing of the interface, and is only meaningful in the interface region where $0<\alpha<1$. The implementation is based on an explicit first-order time integration routine. A detailed description of the solution algorithm can be found in Berberovic et al. (2009). The spatial variation of fluid density and dynamic viscosity can be expressed through the weighting

$$
\begin{aligned}
& \rho=\alpha \rho_{\text {water }}+(1-\alpha) \rho_{\text {air }}, \\
& \mu=\alpha \mu_{\text {water }}+(1-\alpha) \mu_{\text {air }},
\end{aligned}
$$

where the subscripts water and air represent the corresponding fluid property of water and air respectively. Only the laminar flow model is used in the numerical simulations, as the turbulence properties almost generate no effect on the results in this study according to the numerical tests.

\subsection{Numerical implementations and boundary conditions}

The simulations are conducted on the Singapore National SuperComputing Center using OpenFOAM ${ }^{\circledR}$ version 3.0+. The boundary conditions are defined as follows. At the inlet boundary, the velocity is defined as that of a sinusoidal incoming wave and the gradient of pressure is set to zero, while at the outlet boundary the velocity is fixed at zero and the pressure also has a zero gradient. At the bottom of the wave tank and the solid walls of the bodies, no slip boundary condition is applied. The boundary condition for the upper part of the tank is set as 'atmosphere', where the Neumann and Dirichlet types of boundary conditions are prescribed to the velocity and pressure respectively. In this two-phase flow model, the boundary cell face can be in three states which are completely wet in water, completely dry in air, and being intersected by the water surface. For the wet boundary cells, the velocities are prescribed from the chosen wave theory at the cell face centers. The velocities are zero on the dry boundary faces. On the intersected boundary faces (partially wet), the velocities are also prescribed at the center of wet area from the chosen wave theory. For a 2D problem, the boundary condition on the walls in the third dimension is set to 'empty', and for 3D simulations the boundary condition on the normal solid walls is applied in the third dimension.

The pressure-velocity coupling is solved using the PISO (Pressure Implicit with Splitting of Operator) algorithm. A thorough description of the OpenFOAM ${ }^{\circledR}$ discretization schemes in both time and space can be found in Jasak (1996). The largest allowed Courant number is set to 0.25. In cases when it exceeds 
this value for some cells, the time step will be automatically reduced. Time derivatives are approximated by a first-order Euler scheme. Gradients are solved by the Gaussian integration method based on a linear interpolation from cell centers to cell faces. The Gauss Convection-specific schemes are used for the evaluation of divergence terms in the Navier-Stokes equations. When solving for the pressure and velocity, the relative tolerance for their residual is set to $10^{-9}$ and $10^{-8}$, respectively, as the default settings.

\subsection{Wave generation and absorption}

The toolbox 'waves2Foam' is adopted to generate and absorb waves at the boundaries, where two rectangular relaxation zones are defined at the inlet and outlet boundaries (see Fig. 1). Details of the relaxation zone technique can be found in Jacobsen et al. (2012). The following functions are applied within the relaxation zones

$$
\begin{gathered}
\alpha_{R}\left(\chi_{R}\right)=1-\frac{\exp \left(\chi_{R}^{3.5}\right)-1}{\exp (1)-1}, \\
\Phi_{R}=\alpha_{R} \Phi_{R}^{\text {computed }}+\left(1-\alpha_{R}\right) \Phi_{R}^{\text {target }},
\end{gathered}
$$

where $\Phi_{R}$ is either the velocity or volume fraction of water $\alpha$. The definition of $\chi_{R}$ is such that $\alpha_{R}$ is always 1 at the interface between the non-relaxed fluid domain and the relaxation zones, and $\chi_{R} \in[0,1]$. The superscripts computed and target for velocity represent the velocities that calculated in the computational domain and that evaluated or assigned from the chosen wave model, respectively. In the outlet relaxation zone, $\mathbf{u}^{\text {target }}=0$.

\section{2D simulation of side-by-side boxes}

\subsection{Mesh convergence and model validation}

For the purpose of model validation and input parameters calibration, a simple case of two closely positioned, fixed floating boxes in 2D is first considered. Sketch of the numerical model is shown in Fig. 1 . where two identical boxes are placed at the middle of the wave flume and an inlet relaxation zone is located at the left boundary as well as an outlet relaxation zone at the right boundary. Two phase flows, air and water, are modelled. The detailed configuration is as follows. The box breadth is $B=0.5 \mathrm{~m}$, height $H=0.5$ $\mathrm{m}$, draft $D=0.25 \mathrm{~m}$, gap $B_{g}=0.05 \mathrm{~m}$, water depth $h=0.5 \mathrm{~m}$ and air depth $h_{a}=0.5 \mathrm{~m}$. The total length of the wave flume is $18.5 \mathrm{~m}$, with the relaxation zones of two wavelengths each. The origin is defined at the left inlet boundary on the still water level (SWL), with the $y$-axis pointing upwards. This configuration is in accordance with the study of Lu et al. (2008) as well as the experimental tests in Saitoh et al. (2006).

Fig. 2 shows the numerical model generated in OpenFOAM ${ }^{\circledR}$, where the blue color represents the air domain and the red represents the water domain. The built-in mesh generator utility 'blockMesh' is used to discretize this simple geometry domain. Hexahedral cells are utilized in the entire fluid domain. Mesh of higher density is generated around the bodies, especially in the gap. It linearly stretches from the two 


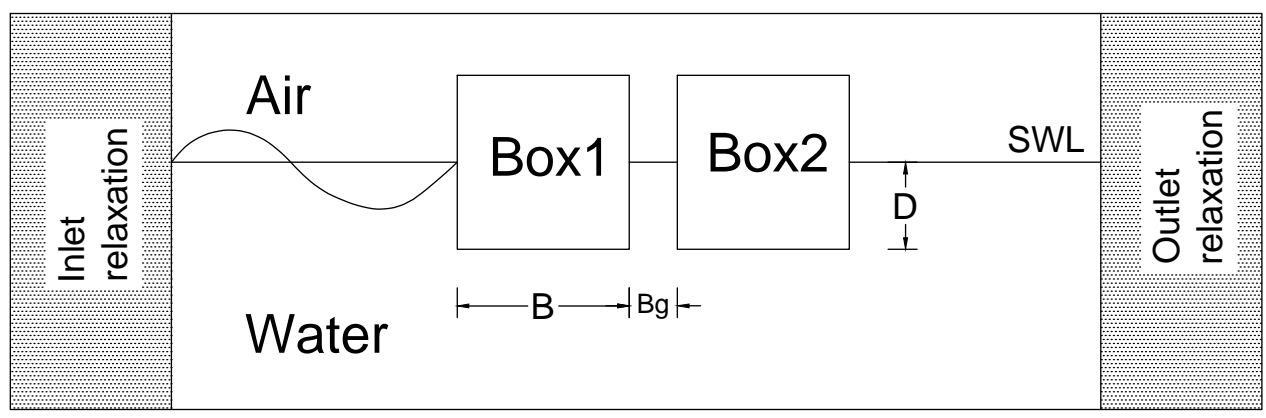

Figure 1: Sketch of numerical wave flume

relaxation zones towards the bodies. Meshes are denser close to the free surface; this is to capture the interface between water and air. A typical computational mesh is shown in Fig. 3.

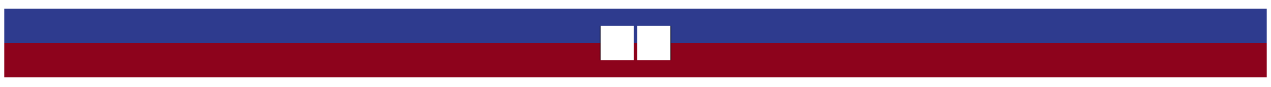

Figure 2: Numerical wave flume with two boxes in OpenFOAM ${ }^{\circledR}$

Mesh convergence study is firstly performed. Three different meshes are generated, namely Mesh 1, Mesh 2 and Mesh 3, which correspond to the coarse, normal and fine meshes respectively. The normal mesh is configured such that the distance of one wavelength in the $x$ direction in the fluid domain composes at least 80 elements and near the free surface the distance of one wave height in the $y$ direction includes at least 20 elements. This requires a very fine mesh in the vertical direction near the still water surface, as shown in Fig. 3(a). This consideration ensures the accurate capturing of the wave profile. Specifically, for Mesh 1 there are 250 elements horizontally distributed in the upstream of the bodies, 45 elements across each body and 15 elements across the gap. Along the height of the bodies 200 elements are adopted. In Mesh 2,360 elements are distributed horizontally in the upstream of the bodies and 300 elements along the body height. The corresponding values are 450 and 400 in Mesh 3. Details of Mesh 1, Mesh 2 and Mesh 3 are listed in Table 1

We consider a case at the critical wave frequency $k h=1.57$ corresponding the gap resonance, where $k$ is the wave number. The incident wave is of $H=0.024 \mathrm{~m}$ height, as used in the experiments in Saitoh et al. (2006). Fig. 4 shows the comparison of wave elevations in the gap for the three mesh configurations. From the plots the three responses are very close. A scrutiny check of the exact values shows about $1 \%$ difference 


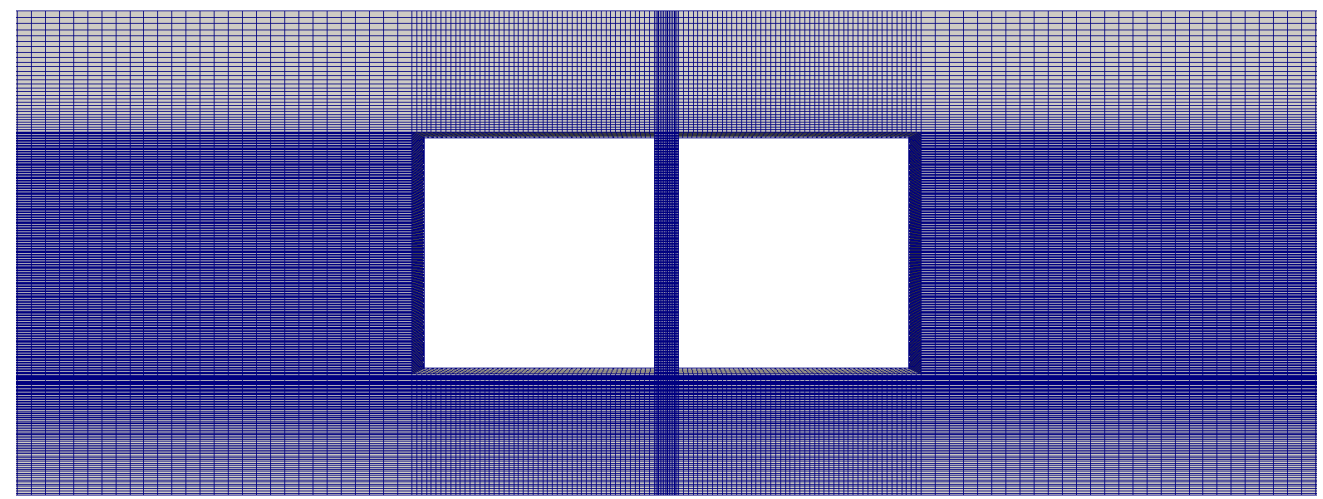

(a)

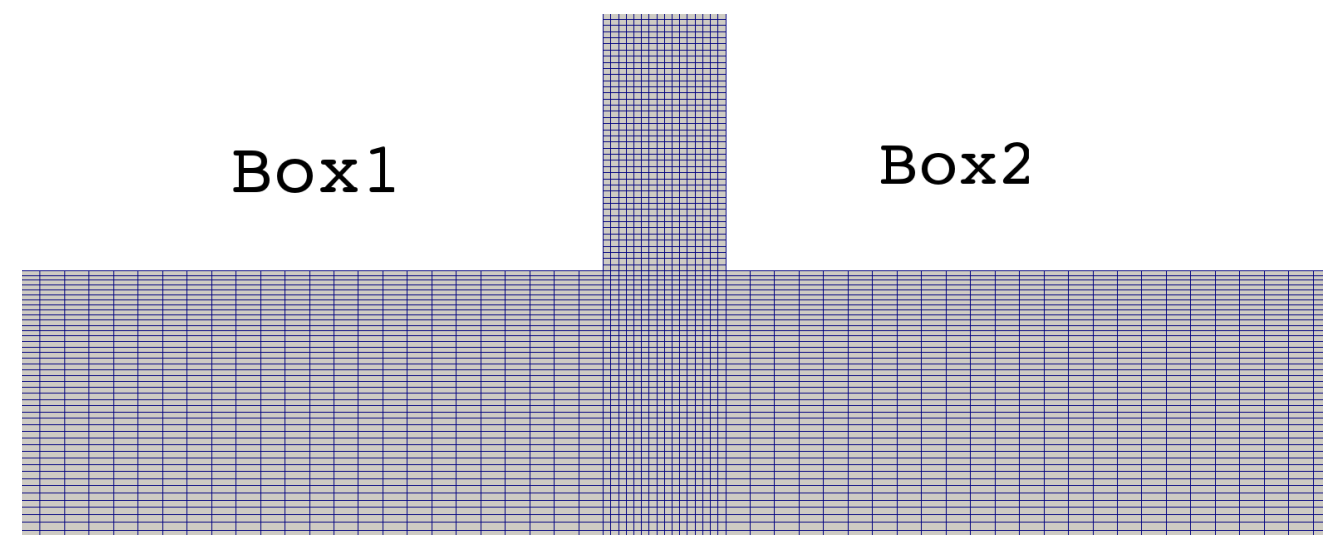

(b)

Figure 3: Computational mesh in the domain: (a) mesh around the boxes; (b) mesh close to the gap inlet

at the wave crest between Mesh 1 and Mesh 2, but almost identical for Mesh 2 and Mesh 3. Considering accuracy and computational effort, in all the following cases, mesh configuration similar to Mesh 2 (normal) is adopted.

Of great concern in this twin-box system is the wave elevation RAOs in the gap. Fig. 5 shows the gap surface RAOs over the non-dimensional wave frequency range $k h=1.0-3.0$. The gap surface elevation is normalized by the incident wave amplitude. There apparently appears a peak response near the frequency $k h=1.57$, where a piston mode of fluid resonance in the gap is observed hence termed as the resonant/critical frequency. Three other results are included for comparison: linear prediction in the gray solid line; viscous flow model results by Lu et al. (2008) in the red dashed line; and experimental measurements by Saitoh et al. (2006) in the black dot. The linear potential flow theory obviously over-predicts the response at the critical frequency, giving a response some 18 times the incident wave amplitude (not shown in the figure). The viscous flow model in Lu et al. (2008) yet somewhat under-estimates the peak response. The present simulation generates predictions most agreeable to the measurements, especially the peak response. Noticeably, all the models can give satisfying predictions at the frequencies other than those near the critical frequency. This 
Table 1: Configurations of three meshes

\begin{tabular}{l|l|l|l}
\hline Cases & No. of cells & No. of points & No. of cell faces \\
\hline Mesh 1 & 135432 & 275864 & 538406 \\
Mesh 2 & 201960 & 406970 & 809366 \\
Mesh 3 & 317060 & 637974 & 1270168 \\
\hline
\end{tabular}

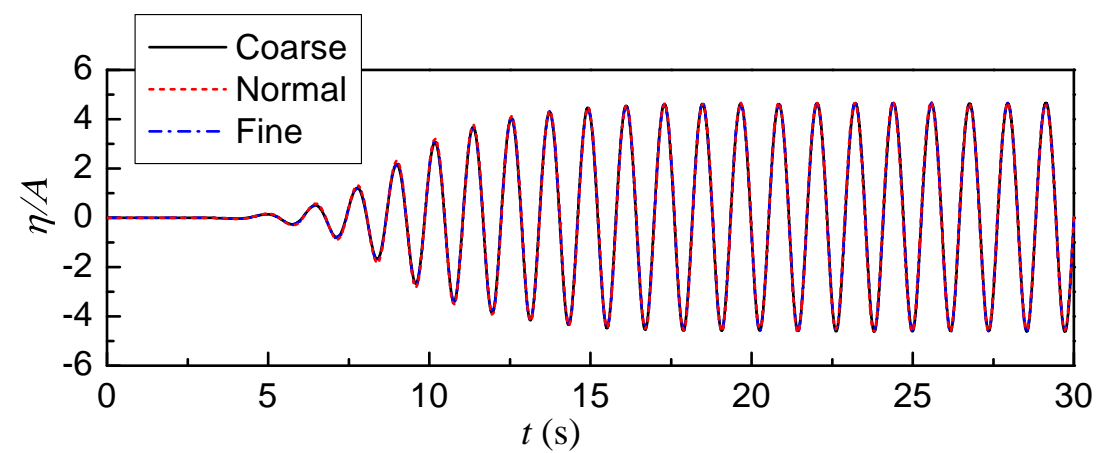

(a)

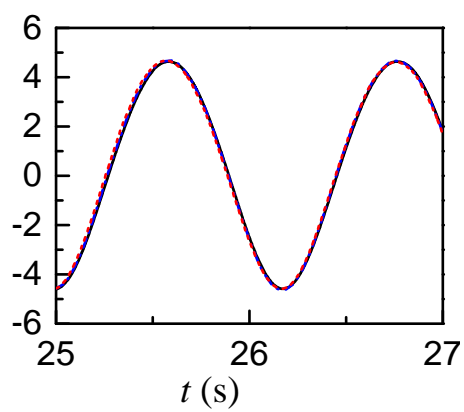

(b)

Figure 4: Grid independence for 2D side-by-side boxes of time histories of wave elevations: (a) full time history; (b) $25-27 \mathrm{~s}$.

simple 2D case demonstrates the accuracy and capability of the model in simulating such wave resonance in a narrow gap formed by two side-by-side floating bodies.

A clear character of the gap resonance is seen in Fig. 6 showing the wave responses at the upwave surface of the upstream box (dashed line $x=8.725 \mathrm{~m}$ ), in the gap (solid line $x=9.25 \mathrm{~m}$ ), and at the leeside of the downstream box (dash-dot line $x=9.775 \mathrm{~m}$ ). The free surface elevation is most significant in the gap, reaching 5 times the incident wave amplitude; the amplification at the front of the upstream box at $x=8.725$ $\mathrm{m}$ is only about 2 times. Minor wave energy has transmitted through the twin-box system. This response variation clearly pictures the occurrence of resonant waves in the gap.

Further comparison can be made for RAOs at other locations. Fig. 7 plots the free surface RAOs at the positions $x=6 \mathrm{~m}, 7 \mathrm{~m}, 12 \mathrm{~m}$, over the frequencies $k h=1-2.5$. It can be seen that the maximum wave response at the locations other than the gap is generally less than 2.5. The RAOs vary between the values 0 and 2, due to the formation of near-standing waves in front of the upstream box. In case of pure reflection, elevations at the anti-nodes of the standing wave would be exactly 2 and at the nodes of the standing wave would be zero. In this case, for $x=6 \mathrm{~m}$ the anti-nodes are expected at $k h=n \pi h /(8.725-6)=0.576 n(n=$ $1,2, \cdots)$ or $1.15,1.73,2.3$; and the nodes at $k h=0.576(n-1 / 2)=1.44,2.02$. For $x=7 \mathrm{~m}$ the corresponding frequencies for the anti-nodes are $k h=0.91,1.82$ and for the nodes $k h=1.37,2.28$. They generally agree with the calculated results in Fig. 7. Unlike the RAO variation in front of the upstream box, the overall 


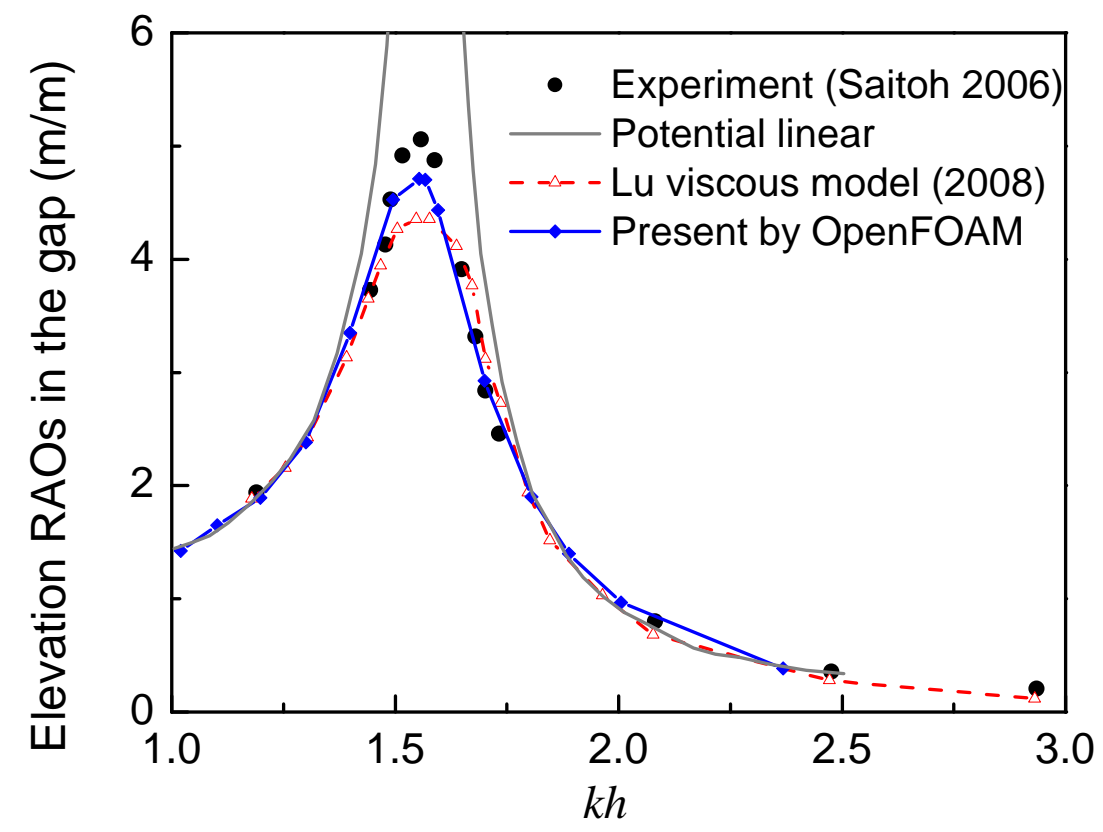

Figure 5: Wave elevation RAOs in the gap with comparisons against linear prediction and experiments

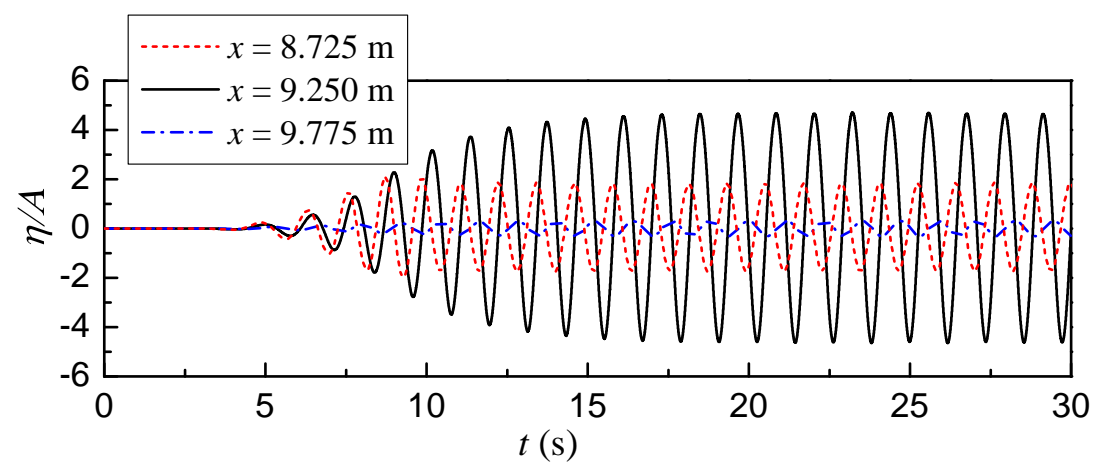

Figure 6: Wave elevation series at different locations at the critical wave frequency $k h=1.57$

RAOs at $x=12 \mathrm{~m}$ behind the downstream box is almost negligible. At the wave frequencies higher than $k h=1.75$, no wave energy is transmitted through the boxes.

\subsection{Flow separation and vortex shedding}

In order to explore the physical reason that potential flow models over-predict the resonant response in the gap, and to demonstrate the flow characteristics inside the gap, we plot the velocity field inside the gap upon resonance in Fig. 8 . The time instant $t=27.7 \mathrm{~s}$ in Fig. 8(a) corresponds to the time when the free water surface in the gap is at its mean level near zero and the fluid is moving upward with the maximum velocity. It shows clearly the flow separation at the gap inlet corners. Meanwhile, a pair of vortices are about to form inside the gap. Surrounding the vortex pair the fluid has the maximum velocity near the gap vertical centerline while near the box surface the velocity is much smaller pointing downward. At the next 


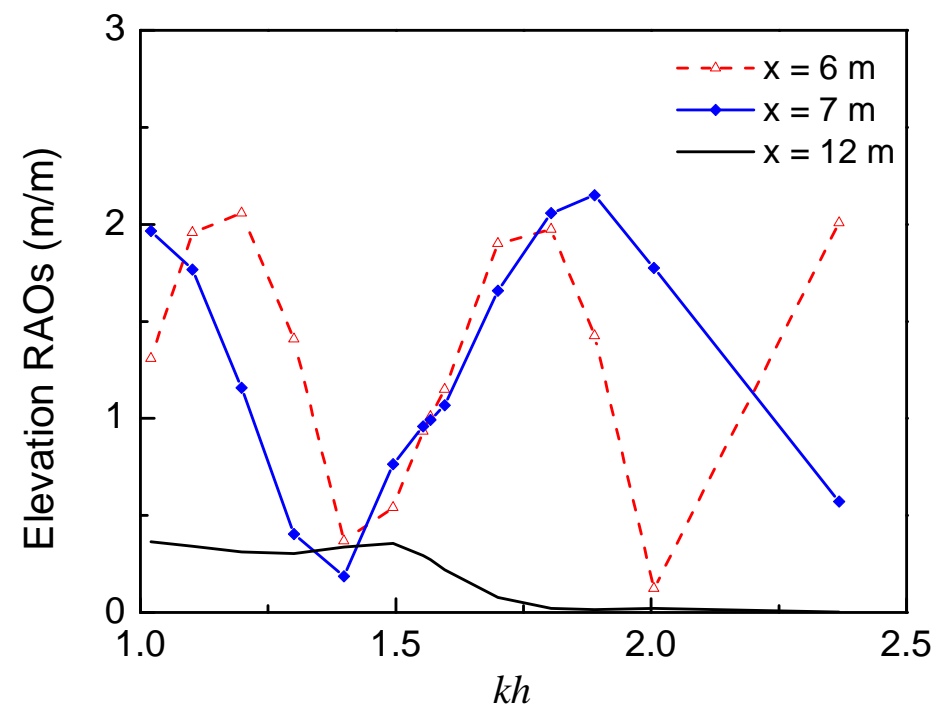

Figure 7: Wave elevation RAOs at locations other than the gap

instant $t=27.8 \mathrm{~s}$ in Fig. 8(b) the vortex pair moves up from the corners following the fluid movement in the gap. Above the vortex pair the velocity distribution is rather uniform across the gap, suggesting the frictional effect on the box surface is insignificant to affect the velocity distribution in the gap. The time instant $t=28.0 \mathrm{~s}$ shows a great drop of velocity magnitude surrounding the vortices. The gap free surface reaches its highest level and most of the fluid near the gap has almost zero velocity, except around the vortex pair. A significant difference now is that the velocity near the vertical surface of the box increases to be greater than the velocity magnitude at the gap vertical centerline. Yet the vortex core position remains the same.

The next time instant $t=28.2 \mathrm{~s}$ in Fig. 8(d) shows a reversed movement of the fluid in the gap, i.e. downward into the main fluid bulk beneath the boxes. At the same time the vortex strength is weakened and the vortices are 'pushed' out of the gap. The following time instant $t=28.4 \mathrm{~s}$ in Fig. 8(e) sees the fading of this vortex pair hence the dissipation of energy associated with the vortices. Nonetheless, there appears another pair of vortices beneath the box corners at $t=28.6 \mathrm{~s}$, with a lower strength than the pair formed in the gap. The left vortex is in the clockwise direction while the other is in the counter clockwise direction, but they are moving towards each other. At $t=28.6 \mathrm{~s}$ the gap free surface reaches its lowest position and the velocity in the gap is close to zero. In the following stages in Figs. $8(\mathrm{~g})$ and $8(\mathrm{~h})$ the fluid in the gap is about to move upward again, and the fluid velocity at the corners will increase and show the higher gradient again. The pair of vortices beneath the boxes disappear and the new pair within the gap is about to build. Again, the velocity at the gap vertical centerline increases and there will start the flow separation at the corners.

The pressure field from $t=27.7 \mathrm{~s}$ to $t=28.9 \mathrm{~s}$ in the fluid near the gap is also plotted to show the flow characteristics in Fig. 9. The pressure plotted is calculated as $p_{h}=p-\rho g h$, where $p_{h}$ is the dynamic 


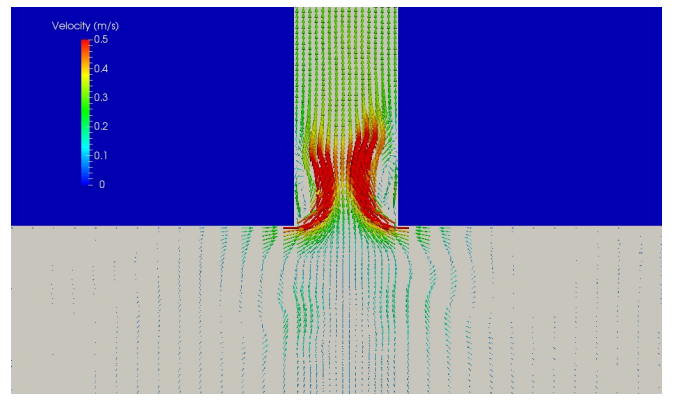

(a) $\mathrm{t}=27.7 \mathrm{~s}$

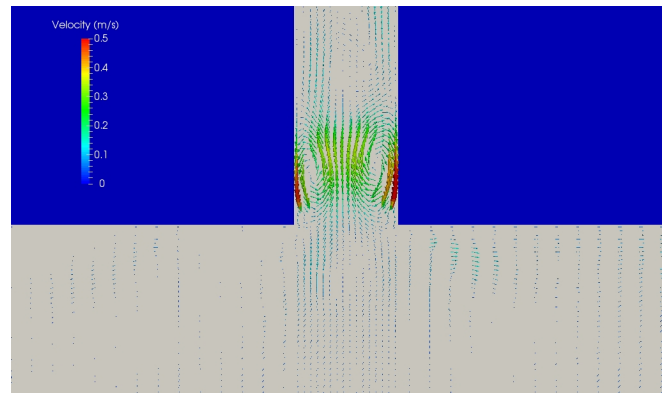

(c) $t=28.0 \mathrm{~s}$

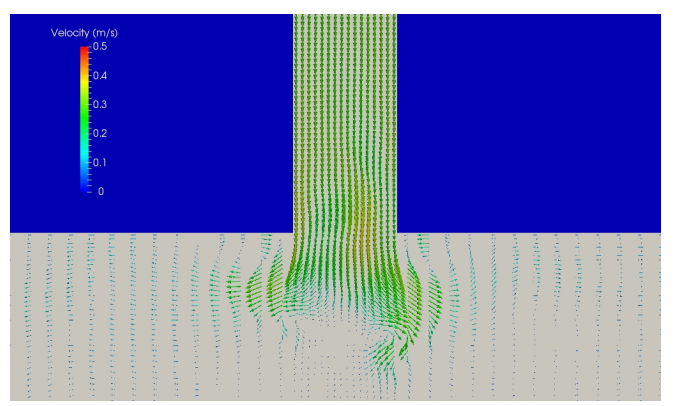

(e) $\mathrm{t}=28.4 \mathrm{~s}$

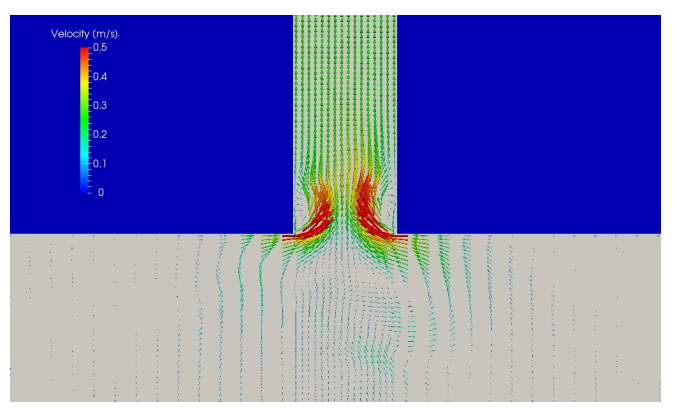

(g) $\mathrm{t}=28.8 \mathrm{~s}$

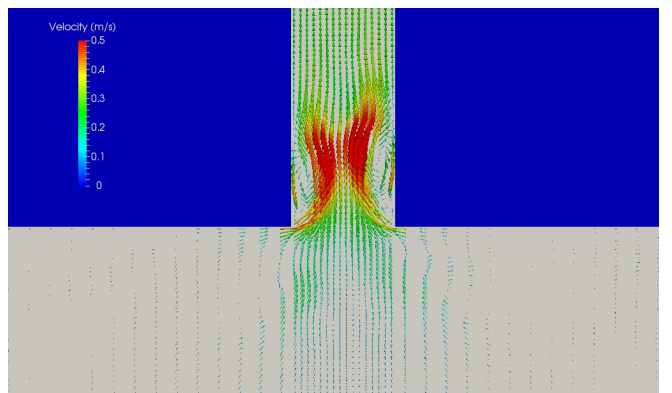

(b) $t=27.8 \mathrm{~s}$

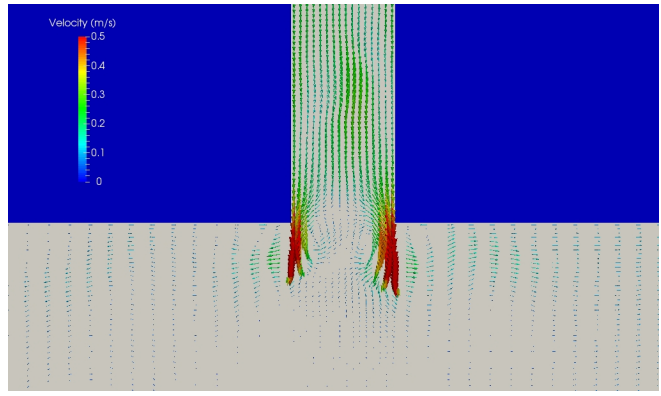

(d) $\mathrm{t}=28.2 \mathrm{~s}$

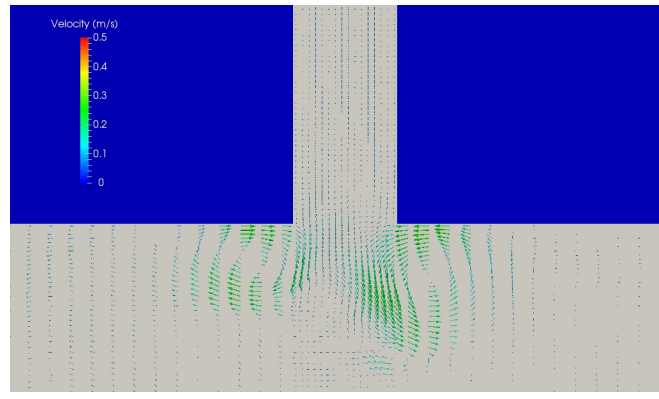

(f) $\mathrm{t}=28.6 \mathrm{~s}$

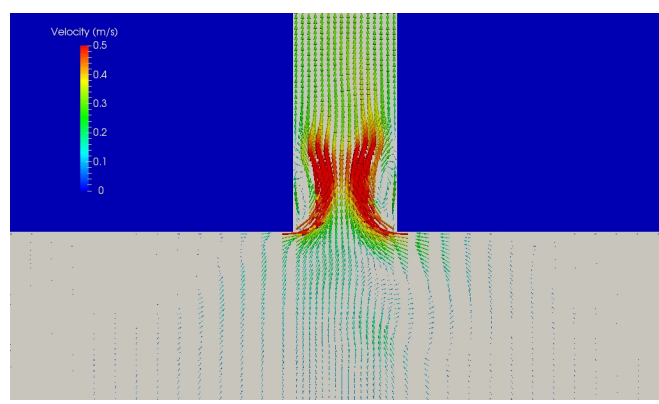

(h) $\mathrm{t}=28.9 \mathrm{~s}$

Figure 8: Velocity vectors around the box corners in the gap at different time instants

pressure. It is obviously seen that the pressure in the vortex region is lower than outside. Taking the time instant $t=27.7 \mathrm{~s}$ in Fig. 9(a) as an example, two low pressure regions just above the box corners inside the 
gap are found, and they coincide with the location of the vortex cores. Comparing with other time instants, this is the moment when the pressure difference between the vortex core and neighbouring regions is the most significant, and it makes the strongest vorticity.

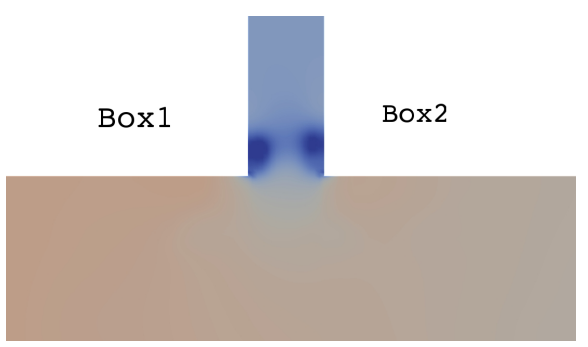

(a) $t=27.7 \mathrm{~s}$

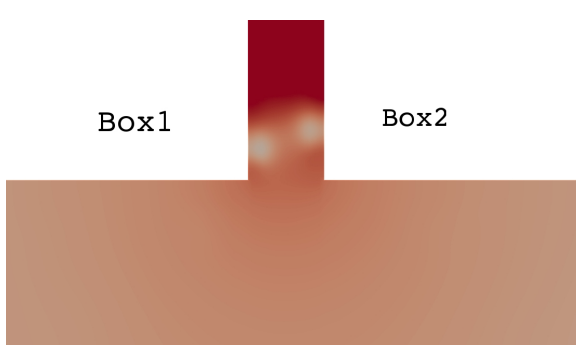

(c) $\mathrm{t}=28.0 \mathrm{~s}$

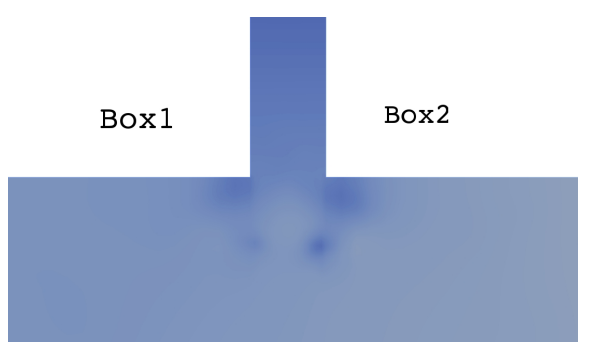

(e) $\mathrm{t}=28.4 \mathrm{~s}$

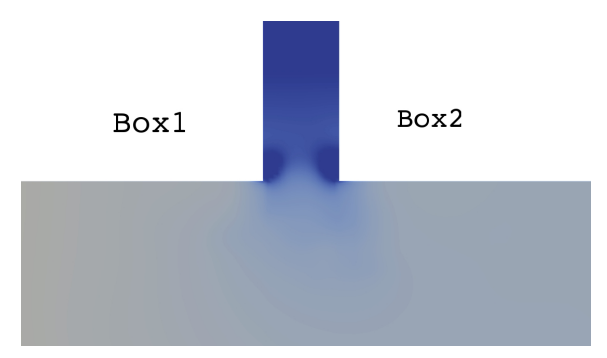

(g) $\mathrm{t}=28.8 \mathrm{~s}$

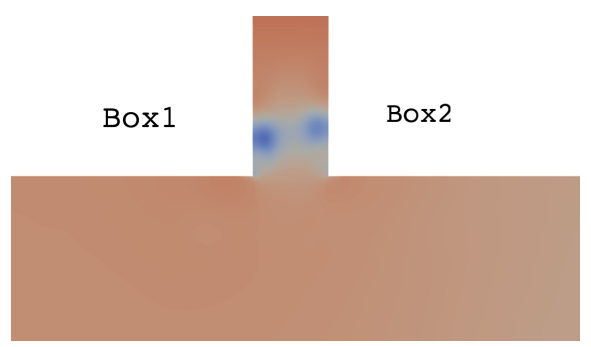

(b) $\mathrm{t}=27.8 \mathrm{~s}$

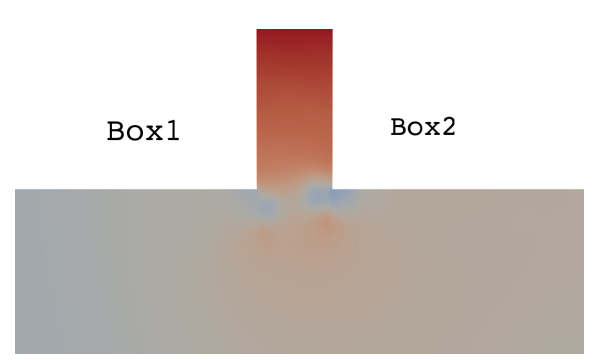

(d) $\mathrm{t}=28.2 \mathrm{~s}$

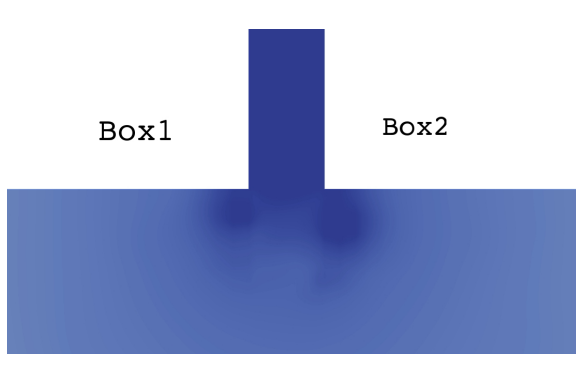

(f) $\mathrm{t}=28.6 \mathrm{~s}$

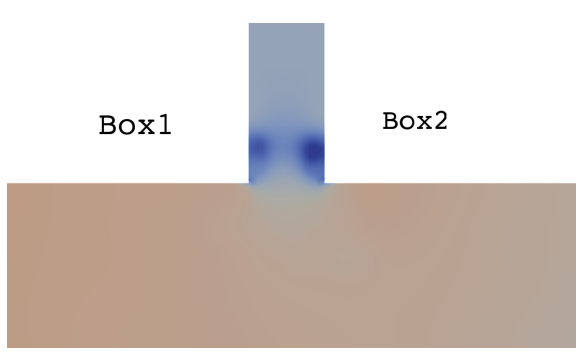

(h) $\mathrm{t}=28.9 \mathrm{~s}$

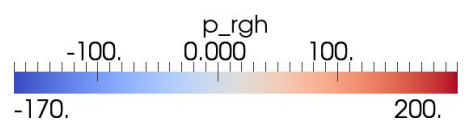

Figure 9: Pressure field around the box corners in the gap at different time instants 
The above process of fluid movement in one wave period demonstrates the formulation and dissipation of vortices around the gap inlet, indicating the strong viscous damping due to the presence of the sharp corners. Such flow separation and vortex shedding process, however, cannot be modelled in any potential flow theory. Therefore, the energy loss due to flow separation and vortex shedding is not taken into account in the linear potential flow model; this well explains the over-estimation of gap resonant response by the linear model in Fig. 5 .

\section{3D simulation of side-by-side barges}

In order to investigate the gap resonance in a more realistic 3D situation, we then simulate two 3D side-by-side barges in a wave basin. This is to consider the fact that the $2 \mathrm{D}$ simulations may provide limited

information on the gap resonance. Two barges of configuration same as that in Molin et al. (2009) are modelled. The side-by-side identical barges float in a $9 \mathrm{~m}$ long, $7 \mathrm{~m}$ wide wave basin. Fig. 10(a) shows the numerical model and meshes in the computational domain. The barges are $2.47 \mathrm{~m}$ long, $0.6 \mathrm{~m}$ wide and $0.335 \mathrm{~m}$ deep. The draft is $0.18 \mathrm{~m}$ and the gap width is $0.12 \mathrm{~m}$. The origin of the coordinate system is located at the center of the narrow gap, on the still water surface, with the $z$ axis pointing upward. The left inlet boundary is $6 \mathrm{~m}$ away from the origin. Again, the blue color represents air and the red is water. The mesh at the air-water interface is extremely dense, to accurately capture the free water surface. Fig. 10(b) shows the mesh around the barges in a sliced plane $y=0.0$. The water depth is $2 \mathrm{~m}$. In order to minimize the number of computational cells, the air depth is set as shallow as only $0.2 \mathrm{~m}$.

\subsection{Mesh convergence}

Similar to the 2D cases, grid dependence needs to be checked before performing further simulations. We test three mesh configurations, namely coarse, normal and fine meshes, of which the normal mesh has a similar mesh density, i.e. at least 80 elements along one wave length and 20 elements along one wave height near the free water surface, as the 'standard' configuration defined for the $2 \mathrm{D}$ cases. Numbers of cells, points and cell faces in each mesh configuration are listed in Table 2. We consider the most critical case near the resonant frequency. Only the beam sea cases are considered in this study. The potential flow model in Feng and Bai (2015) predicted the gap resonance (piston mode) occurring at $\omega=5.75 \mathrm{rad} / \mathrm{s}$, however, the CFD model in the following section predicts the resonant frequency at $\omega=5.70 \mathrm{rad} / \mathrm{s}$. Here we use $\omega=5.70$ for the model convergence study. The incoming wave height is firstly set as $0.01 \mathrm{~m}$. Both the inlet and outlet relaxation zones occupy a length of 1.5 times the wave length. Of interest is the wave elevation at the middle of the narrow gap. Fig. 11 plots the time histories of normalized wave elevations at the mid-gap for 30 seconds, for the three mesh configurations. A clear character of the near-resonance response is the slow build-up of the elevation amplitude. The final steady state is only achieved after 25 seconds. Wave elevations for the three cases essentially are very close, except at the maxima at the steady state. It reveals that there exists some $5 \%$ difference between the coarse mesh and the other two at the maxima, while the normal and 


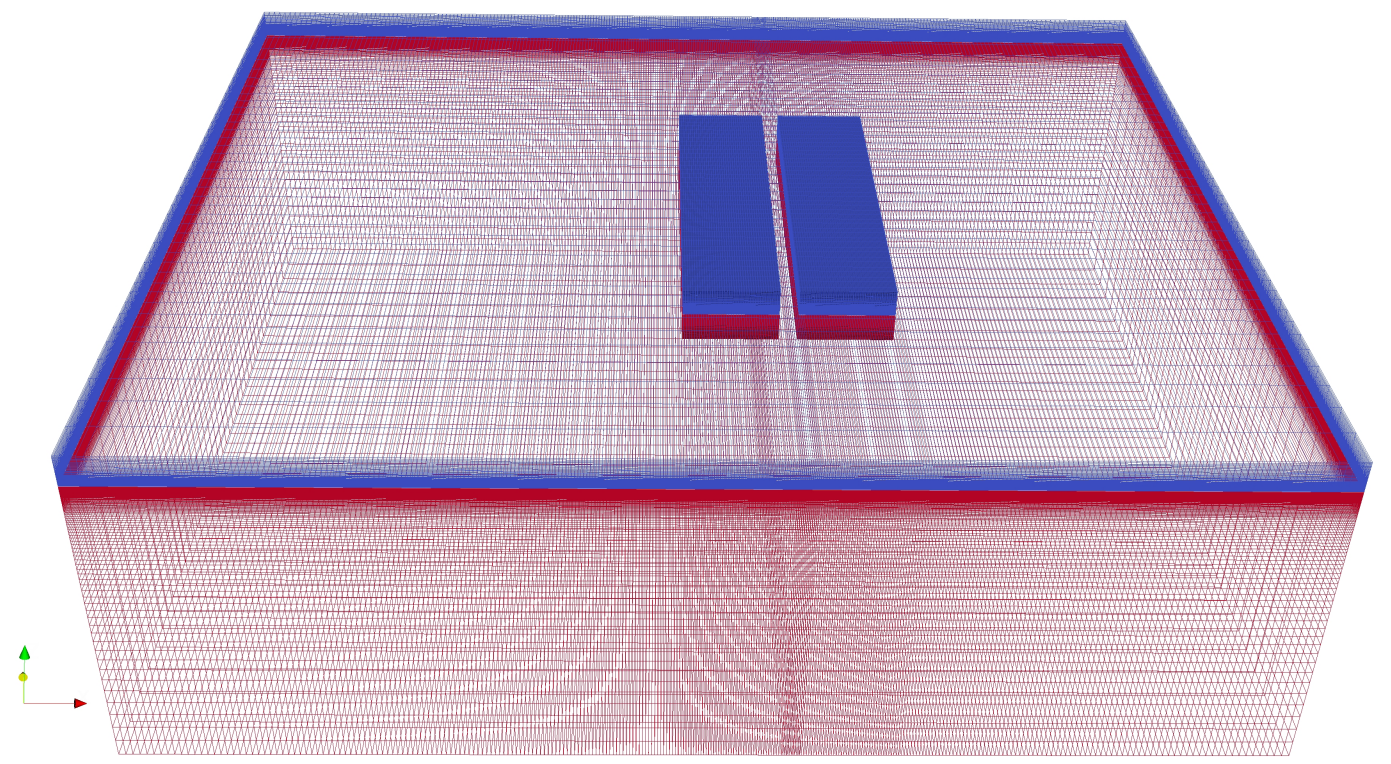

(a) 3D barges mesh

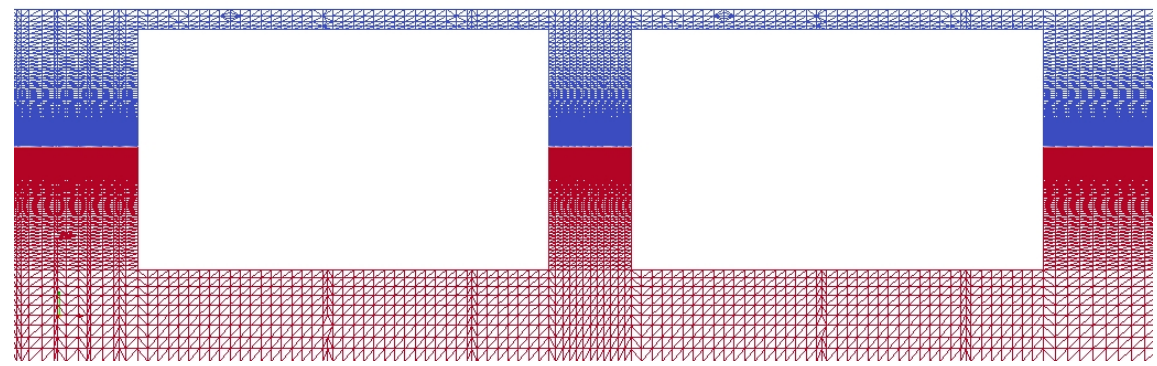

(b) Mesh around barges at mid-gap

Figure 10: 3D model of side-by-side barges in a wave basin

fine meshes are almost identical. This grid dependence study suggests that the normal mesh configuration is sufficiently fine (to save computational time) for the model here, hence is adopted in the rest of the study.

\subsection{Incoming wave calibration}

The accuracy of modelling wave interaction with floating bodies relies on the accurate generation of incoming waves. Calibration of incoming waves is conducted by running a wave tank of exactly the same size as the model studied but without bodies, by which the significance of the numerical diffusion can be detected. Input parameters of the incoming wave are kept the same, i.e. $\omega=5.70 \mathrm{rad} / \mathrm{s}$ and $H=0.01 \mathrm{~m}$. We put the wave probes along the centerline of the tank $(x=-6.0 \mathrm{~m}-3.0 \mathrm{~m} ; y=0.0 \mathrm{~m})$. Fig. 12 shows the wave elevations at three locations: $x=-4.0 \mathrm{~m}$ where the inlet relaxation zone just ends, $x=-0.67$ 
Table 2: Configurations of three meshes for 3D model

\begin{tabular}{l|l|l|l}
\hline Mesh & No. of cells & No. of points & No. of cell faces \\
\hline Coarse & 1105040 & 1175662 & 3285646 \\
Normal & 2126720 & 2227027 & 6480368 \\
Fine & 3423550 & 3520342 & 9302682 \\
\hline
\end{tabular}

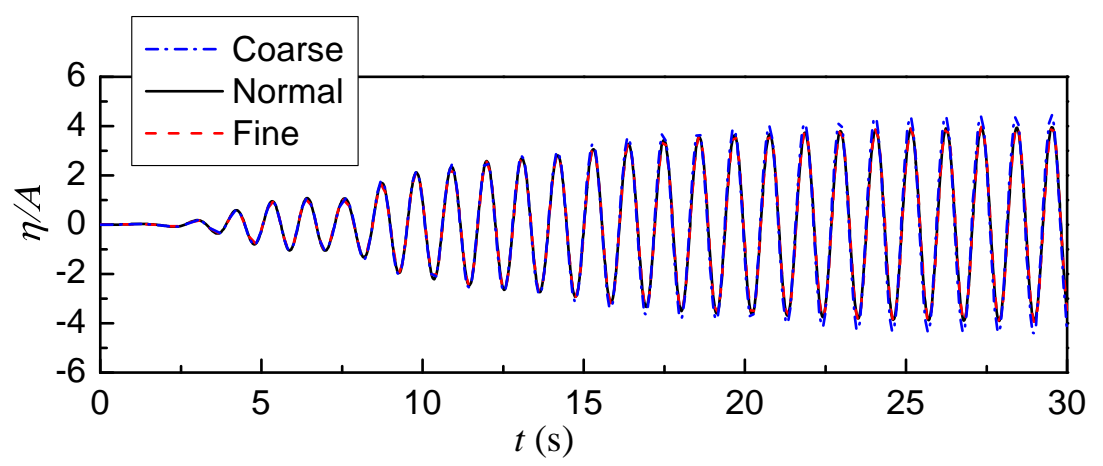

Figure 11: Wave elevations at the mid-gap for three different mesh configurations with wave frequency $\omega=5.70$

$$
\mathrm{rad} / \mathrm{s}
$$

$\mathrm{m}$ upfront the floating barges in the corresponding case with bodies, and $x=0.0 \mathrm{~m}$ at the gap center. The elevation amplitude at $x=-4.0 \mathrm{~m}$ reaches the targeted value very quickly within $2 \mathrm{~s}$. The other two locations also reach the non-dimensional amplitude $(\eta / A=1)$ after about $12 \mathrm{~s}$.

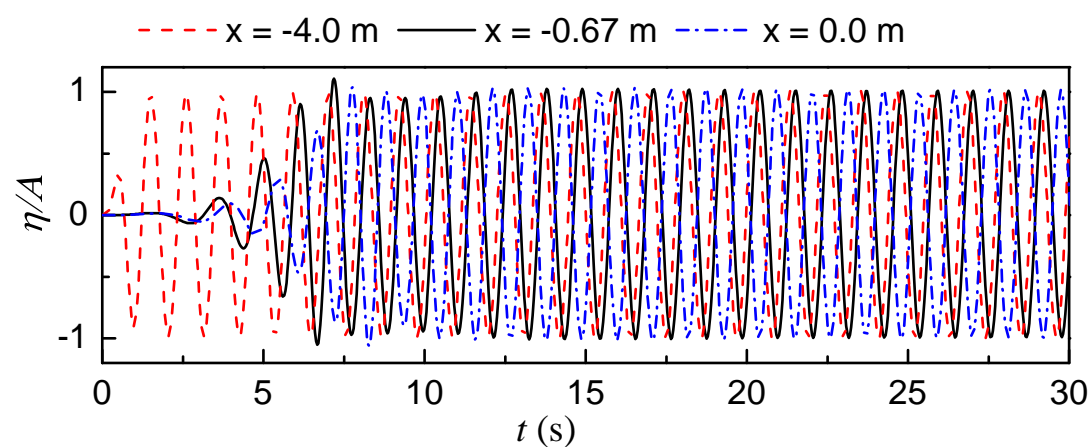

Figure 12: Wave elevations at different locations in a wave tank without bodies at $\omega=5.70 \mathrm{rad} / \mathrm{s}$

The wave profile can be examined as well. Fig. 13 shows the wave profile along the centerline $y=0$ of the tank without bodies at three time instants. The incoming waves maintain almost the constant amplitude when reaching $x=0.0 \mathrm{~m}$, although small variation is observed among the three time instants. Further, the outlet relaxation zone is demonstrated effective in preventing wave reflection from the outer boundary at $x=3 \mathrm{~m}$. The testing of pure wave generation in the tank is to ensure that when the incoming wave reaches the floating bodies it is indeed our targeted incident wave. This wave generation study again shows that the 
inlet/outlet relaxation techniques generate high quality waves for the further simulation of wave interaction with bodies.

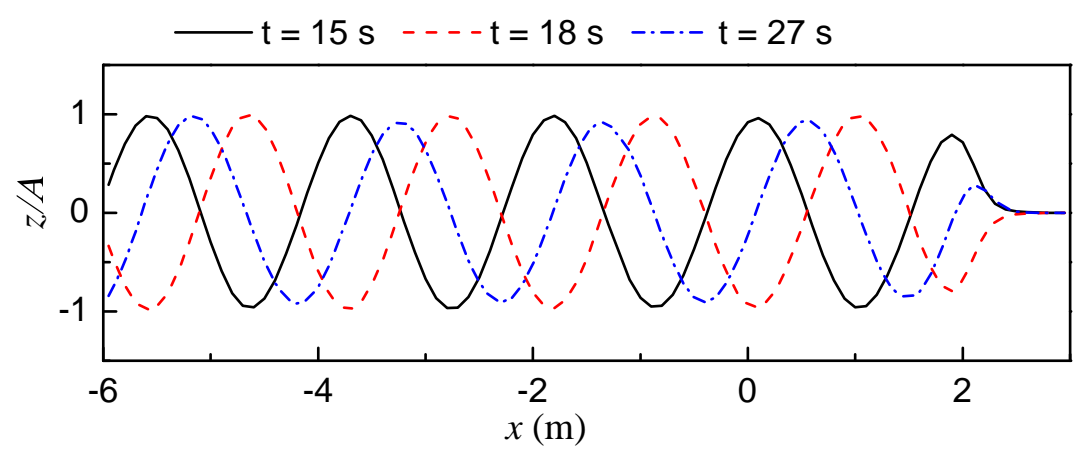

Figure 13: Wave profiles along the centerline of a wave tank without bodies at three different time instants

\subsection{Simulation results}

We first keep the incident wave height as $0.01 \mathrm{~m}$ and the frequency $5.70 \mathrm{rad} / \mathrm{s}$. In the following CFD simulations, we will show that the frequency $5.70 \mathrm{rad} / \mathrm{s}$ corresponds to the piston resonant mode. Note that the CFD model predicts a slightly lower piston mode frequency than the linear potential flow model.

To demonstrate the 3D piston resonant mode in the gap at the frequency $5.70 \mathrm{rad} / \mathrm{s}$, we extract the free water surface (the volume fraction $\alpha=0.5$ ) in the entire domain at each output step. Fig. 14 shows the non-dimensionalized wave elevations at four time instants: $\mathrm{t}=38.4 \mathrm{~s}, 38.7 \mathrm{~s}, 38.9 \mathrm{~s}$ and $39.2 \mathrm{~s}$. Fig. 14(a) corresponds to the moment when the gap free surface is at its mean position. The water surface is almost horizontal along the entire gap. The water column in the gap is 'pumped' up at $t=38.7 \mathrm{~s}$ in Fig. 14 (b) and the gap surface returns to the still water level again at $\mathrm{t}=38.9 \mathrm{~s}$ in Fig. 14(c). Moving further downwards, the gap surface reaches the lowest position at $\mathrm{t}=39.2 \mathrm{~s}$ in Fig. 14(d). It should be noted that the timedomain potential flow models are also able to capture the basic characteristics of the gap resonance as well as the resonant mode shapes, see details in Feng and Bai (2015).

Here a major value of CFD simulations is to understand the viscous damping mechanism within the narrow gap. The velocity distribution is of great importance for inspecting the fluid movement. We plot the distribution of velocity magnitude in the water in the vicinity of the gap in Fig. 15 (again only in the $y=0$ plane). The movement of water in one wave period is demonstrated. Starting from the time instant $t=38.4$ $\mathrm{s}$ when the gap free surface is at its mean position $(z=0)$, the free surface actually is at its maximum velocity. We see the water beneath the barges enter the gap, showing a clear contraction effect. The velocity direction at the barge sharp bilges (corners) is about 45 degrees pointing to the gap vertical centerline. With such movement, there forms two low velocity zones in the gap just above the bilges. Near the gap free surface the velocity is almost uniform across the gap (no translateral sloshing). The contraction effect is weakened at $t=38.5 \mathrm{~s}$ with a lower velocity in the gap. At this moment, the fluid close to the vertical walls of the barges at $x= \pm 0.06 \mathrm{~m}$ still has an upward velocity. 


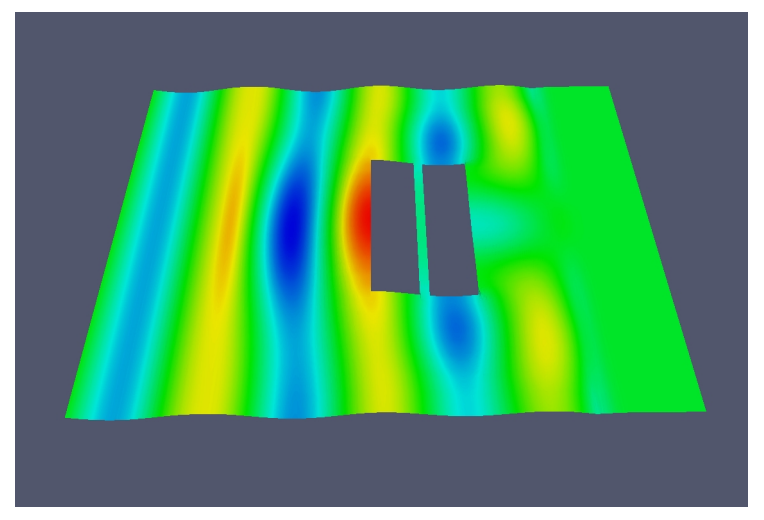

(a) $\mathrm{t}=38.4 \mathrm{~s}$

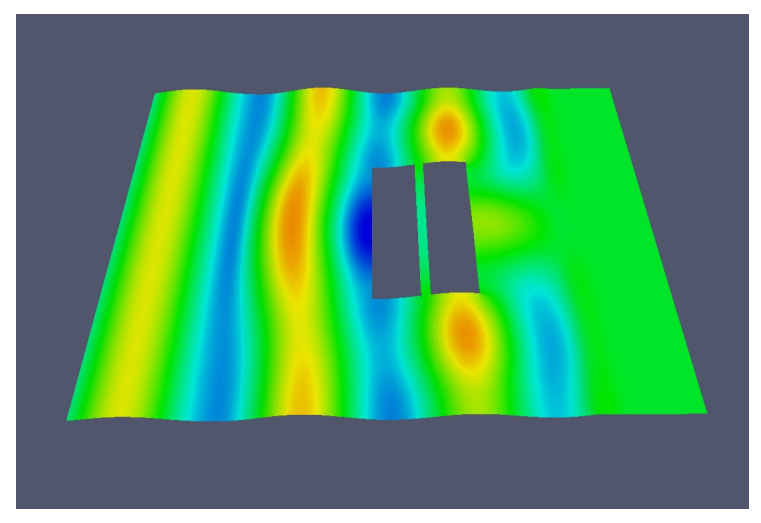

(c) $\mathrm{t}=38.9 \mathrm{~s}$

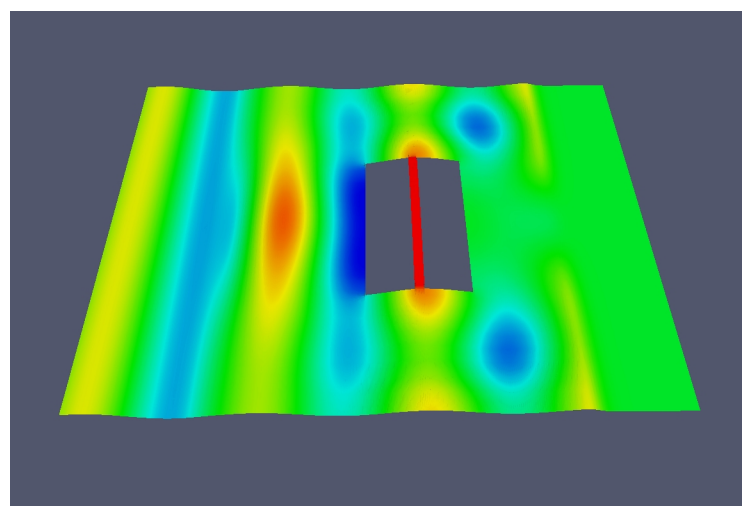

(b) $\mathrm{t}=38.7 \mathrm{~s}$

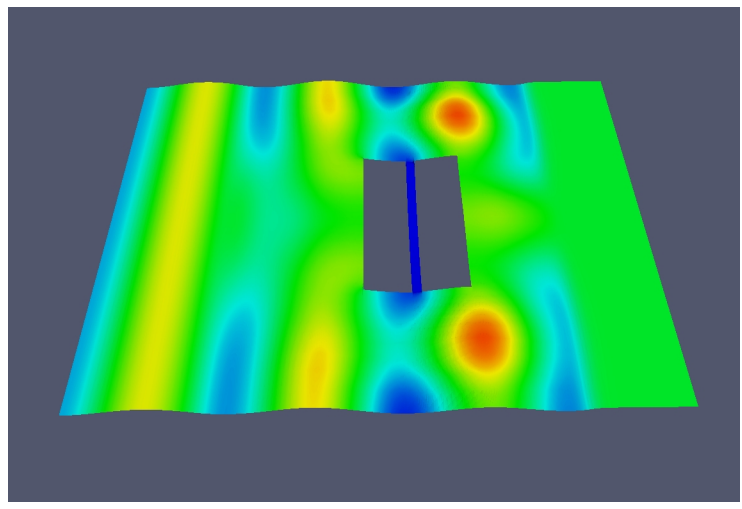

(d) $\mathrm{t}=39.2 \mathrm{~s}$

Figure 14: Free surface elevations for $H=0.01 \mathrm{~m}, \omega=5.70 \mathrm{rad} / \mathrm{s}$ at four different time instants

The next time instant $t=38.6 \mathrm{~s}$ shows a well developed vortex pair in the gap, and the fluid near the barge walls $x= \pm 0.06 \mathrm{~m}$ starts to move downwards. At the time instant $t=38.7 \mathrm{~s}$, the downward velocity near the barge walls is increased while the fluid at the gap center $x=0 \mathrm{~m}$ is almost stagnant. Now the vortex pair is closer to each other, about to contact; and this is the moment when the gap free surface reaches its highest level. In the following time instants $t=38.8-39.1 \mathrm{~s}$, we see the fluid in the gap move out of the gap and the vortex pair merges and dissipates into the main fluid domain. When the gap free surface reaches its lowest level at $t=39.2 \mathrm{~s}$, a larger vortex (of a lower strength) forms and fills up the gap, and the velocity at the barge corners is -45 degrees pointing downwards. The gap fluid starts moving up again in the following time instants when the large vortex dissipates. The above process repeats every wave period and a large amount of energy seems to transform to the thermal energy through the vortices dissipation. This process of flow separation, and vortex formulation and dissipation reasonably reflects as the viscous damping in a broader view of wave interaction with the two barges. It should be mentioned that the velocity field displayed above is at the mid-gap station $(y=0.0 \mathrm{~m})$ where the elevation and velocity are the highest along the gap. From the mid-gap to the gap ends, the velocity reduces and the flow separation becomes less 
significant, resulting in a reduced viscous damping effect. This is the main reason that the potential flow prediction in $3 \mathrm{D}$ situations is more reliable than that in $2 \mathrm{D}$ cases.

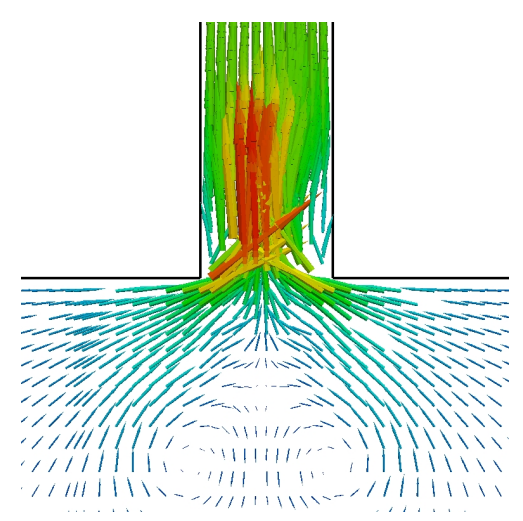

(a) $\mathrm{t}=38.4 \mathrm{~s}$

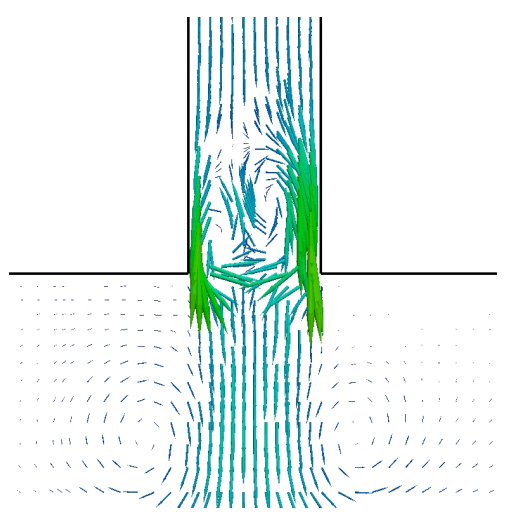

(d) $\mathrm{t}=38.7 \mathrm{~s}$

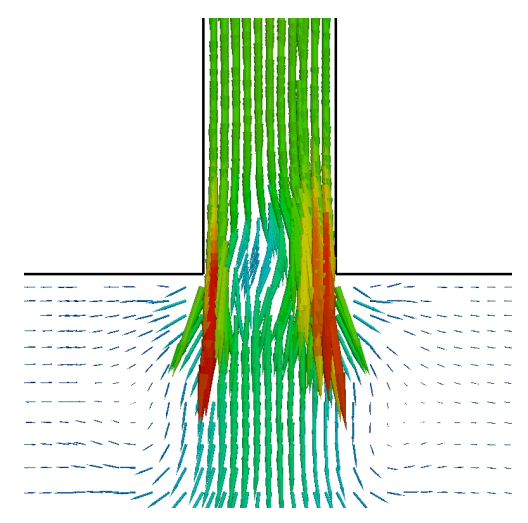

(g) $\mathrm{t}=39.0 \mathrm{~s}$

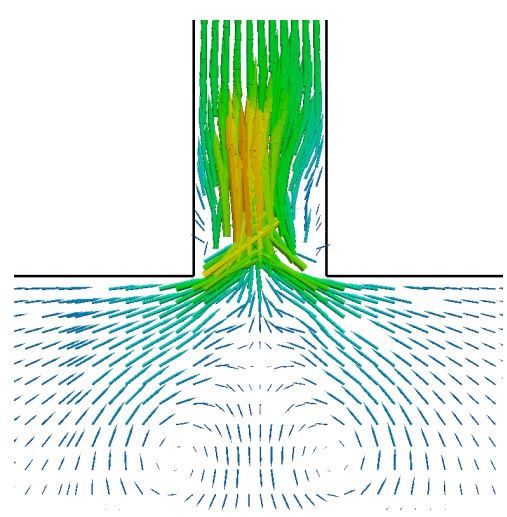

(b) $\mathrm{t}=38.5 \mathrm{~s}$

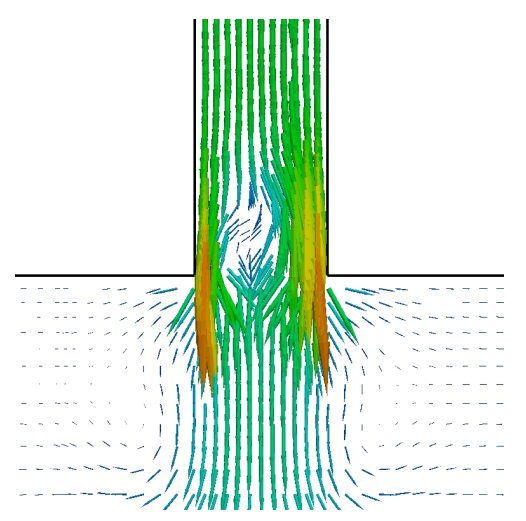

(e) $\mathrm{t}=38.8 \mathrm{~s}$

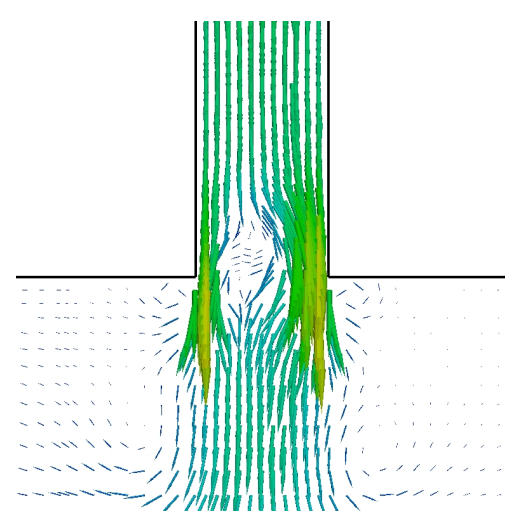

(h) $\mathrm{t}=39.1 \mathrm{~s}$

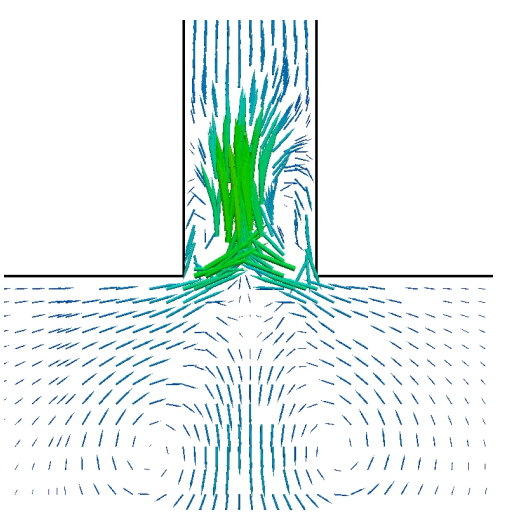

(c) $\mathrm{t}=38.6 \mathrm{~s}$

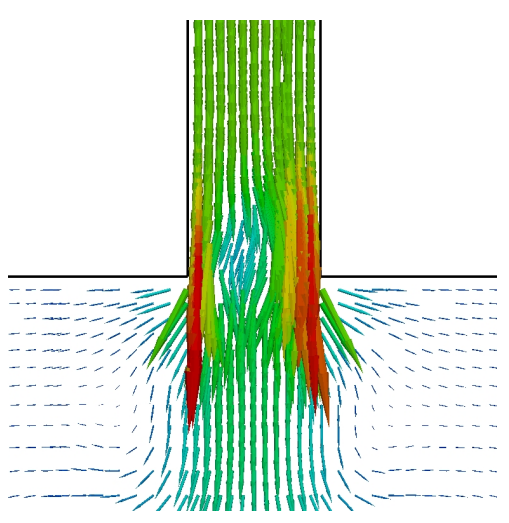

(f) $\mathrm{t}=38.9 \mathrm{~s}$

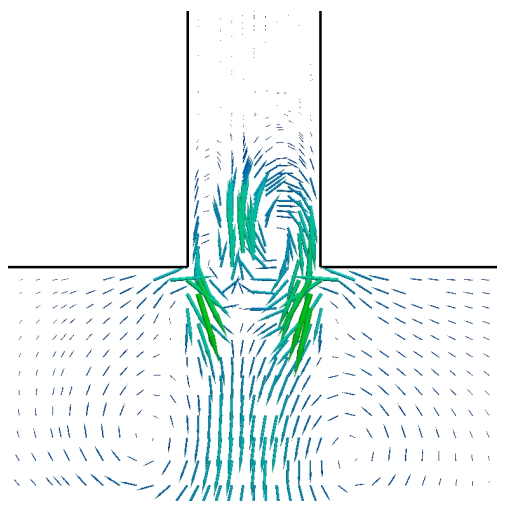

(i) $\mathrm{t}=39.2 \mathrm{~s}$

Figure 15: Velocity vectors around the barges of square bilge in the gap at different time instants 
To demonstrate the 3D view of flow characteristics upon gap resonance, we plot the velocity streamlines at four moments in Fig. 16. The streamlines initiate from two straight lines parallel to (0.02 $\mathrm{m}$ away) the two barge bilges and stretch in both the local velocity direction and the opposite direction. The number of streamlines is 50 along each straight line. Figs. 16(a) and 16(c) correspond to the largest velocities at the bilges. Although flow separation at the bilges starts to occur at this moment, no vortex has been formed. It shows a nearly 2D distribution through the gap except at the two ends where the flow is also 'pumped' in/out of the openings. Figs. 16(b) and 16(d) correspond to the moments when the gap free surface is at its highest and lowest levels respectively, with the near zero velocities but the vortices are most significant near the bilges. The streamlines show high concentration along the gap, indicating the rotational flows in the gap. Again the end effect can be observed.

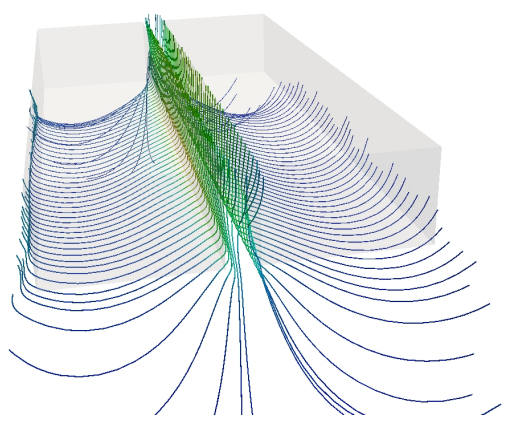

(a) $\mathrm{t}=38.4 \mathrm{~s}$

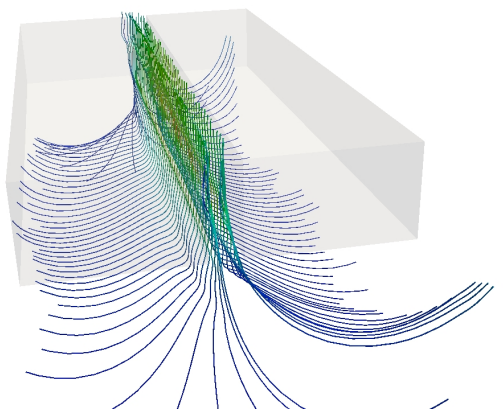

(c) $\mathrm{t}=38.9 \mathrm{~s}$

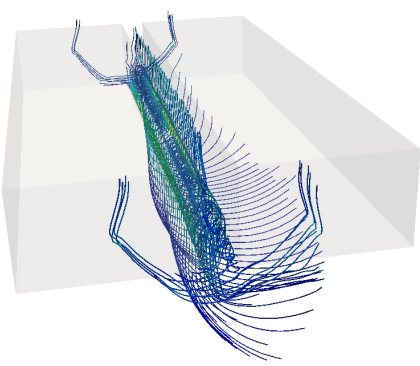

(b) $\mathrm{t}=38.7 \mathrm{~s}$

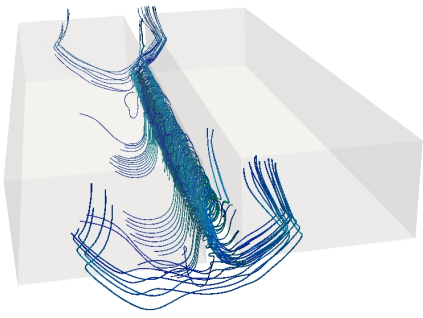

(d) $\mathrm{t}=39.2 \mathrm{~s}$

Figure 16: 3D velocity streamlines around the barges of square bilge

The vorticity iso-surface distribution at the time $t=38.6 \mathrm{~s}$ is further displayed in Fig. 17. It is clear that the vortices are formed around the four barge corners. The vortex pairs within the gap and beneath show stronger strength due to the amplified resonant movement of fluid. Note that vortices are also formed at the two corners away from the gap inlet, though their strengths are rather insignificant. The development and dissipation of the vortices indicate a possible source of damping due to the viscosity of fluid, which is however not modelled in any potential flow model. 


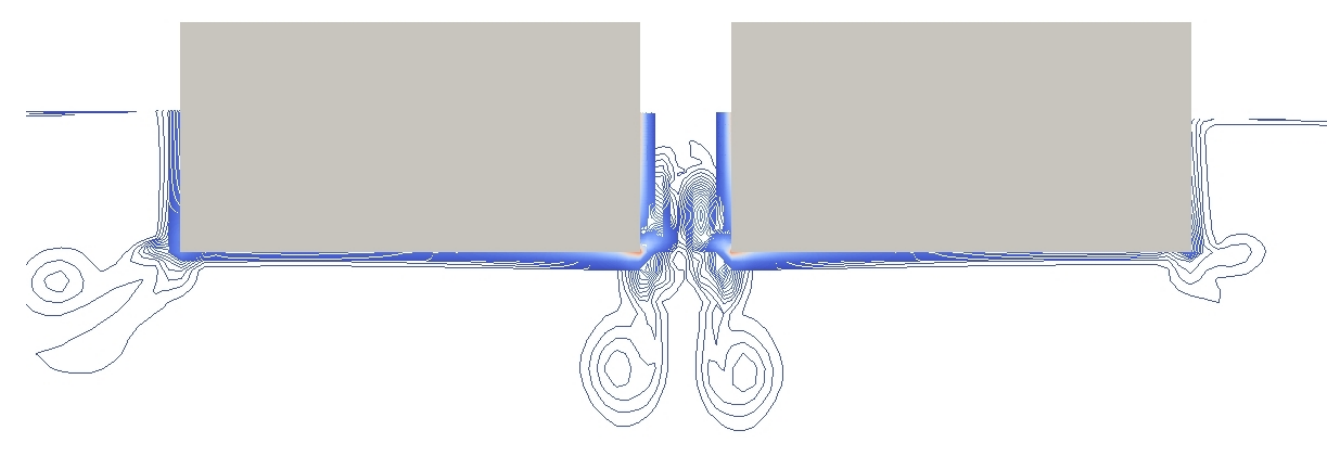

Figure 17: Vorticity iso-surface around the gap at the station $y=0$ at the time instant $t=38.6 \mathrm{~s}$

To demonstrate the viscous effect on the gap resonant response, we perform a series of simulations at different incident wave frequencies near the resonant frequency. Fig. 18 shows the free surface RAOs obtained by the CFD simulations at the middle of the gap $(0,0)$ over the frequency range $5-7.5 \mathrm{rad} / \mathrm{s}$ for the wave height $0.01 \mathrm{~m}$. Included for comparison are the linear potential flow model prediction and the experimental data in Molin et al. (2009). Note that the measurements are extracted from the irregular wave tests by the cross spectral analysis. It shows that the present CFD simulation generates much improved prediction for the gap surface RAOs especially around the first peak, given that the linear model over-predicts both the first and second peaks near $5.70 \mathrm{rad} / \mathrm{s}$ and $6.70 \mathrm{rad} / \mathrm{s}$ respectively. At the frequencies other than the peak response regions, all models can give agreeable predictions. It was illustrated in Feng and Bai (2015) that the nonlinear potential flow model cannot reduce the over-predicted peak responses from the linear predictions. A major difference between the potential flow models and the CFD model is that the CFD model accounts for the viscous effect within the fluid domain. At the gap resonance, the present CFD simulations have shown the formation of vortices and their dissipation around the barge bilges. This suggests that the viscous damping plays a major role in reducing the gap resonant responses, while the nonlinear effect due to the nonlinear free surface boundary conditions is minor. It should be noted that in Molin's tests, the irregular waves were adopted with the significant wave height $H_{s}=0.02 \mathrm{~m}$ and wave period $T_{p}=1 \mathrm{~s}$, rather than the regular waves used in the present study.

\subsection{Effect of wave steepness}

In order to further assess the damping effect due to the fluid viscosity in the gap resonance, we perform CFD simulations using various incident wave amplitudes, as the viscous damping is subject to the wave oscillation amplitude. With the focus on the piston mode only, simulations over the frequency range 5.5 - $5.9 \mathrm{rad} / \mathrm{s}$ are carried out for the wave heights of $H=0.02 \mathrm{~m}, 0.04 \mathrm{~m}$ and $0.05 \mathrm{~m}$. The surface RAOs at the middle of the gap are compared in Fig. 19. According to the figure the RAO shows an asymptotic performance against the wave height, and further increasing the height most probably does not vary the RAO 


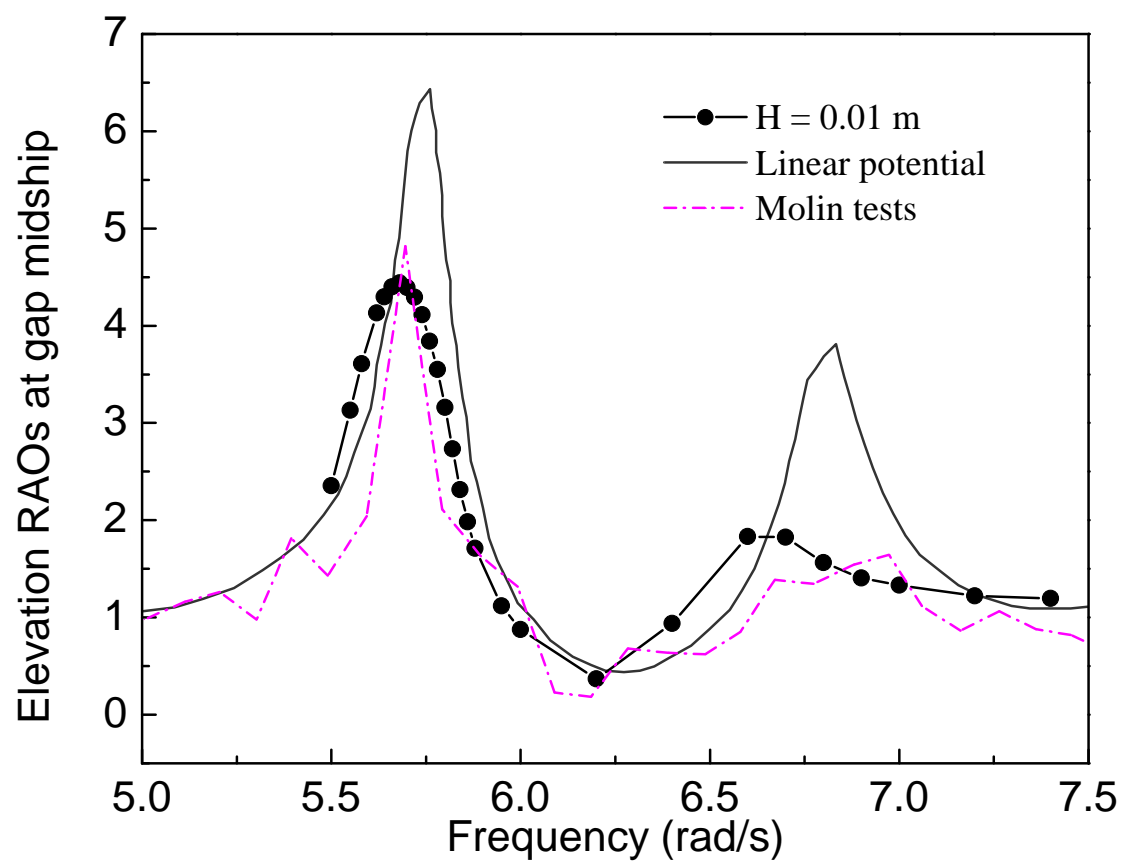

Figure 18: Comparison of free surface RAOs at the mid-gap between linear model, CFD and measurements

noticeably. It is seen that with the increase of wave steepness/height the gap surface RAOs are dramatically reduced. Linear prediction is independent of wave steepness, while the CFD calculation reveals that the wave amplitude plays a critical role for the gap resonant responses. As can be seen, the peak RAO reduces from 4.5 for $H=0.01 \mathrm{~m}$ to as low as 2.1 for $H=0.05 \mathrm{~m}$. Another important feature of RAOs for steeper waves is the broader frequency range for the resonance, which makes the prediction of resonant frequency insensitive compared with the sharp RAOs by the linear model.

The fact of the higher damping ratio induced by steeper waves may be explained by the damping mechanism demonstrated above. With higher wave heights, the vertical fluid velocity on the free surface is increased. Hence the fluid near the barge bilges enters/leaves the gap much faster, creating severe separation hence stronger vortices near the barge bilges. With larger circulation induced, more kinetic energy is transmitted to thermal energy through vortex dissipation. However, the energy dissipation due to fluid viscosity cannot be taken into account in potential flow models, resulting in steepness-independent prediction of RAOs. The present study suggests a strong relationship between the system viscous damping and the incident wave steepness. In the linear potential flow models which incorporate the artificial damping coefficient, the incident wave steepness should be an important consideration in selecting an appropriate damping coefficient.

\subsection{Barges of rounded bilges}

To confirm the damping mechanism, we now simulate the same side-by-side barges with rounded bilges at the gap inlet. The radius of bilge curvature is set to be small $(R=0.04 \mathrm{~m})$ such that the volume of 


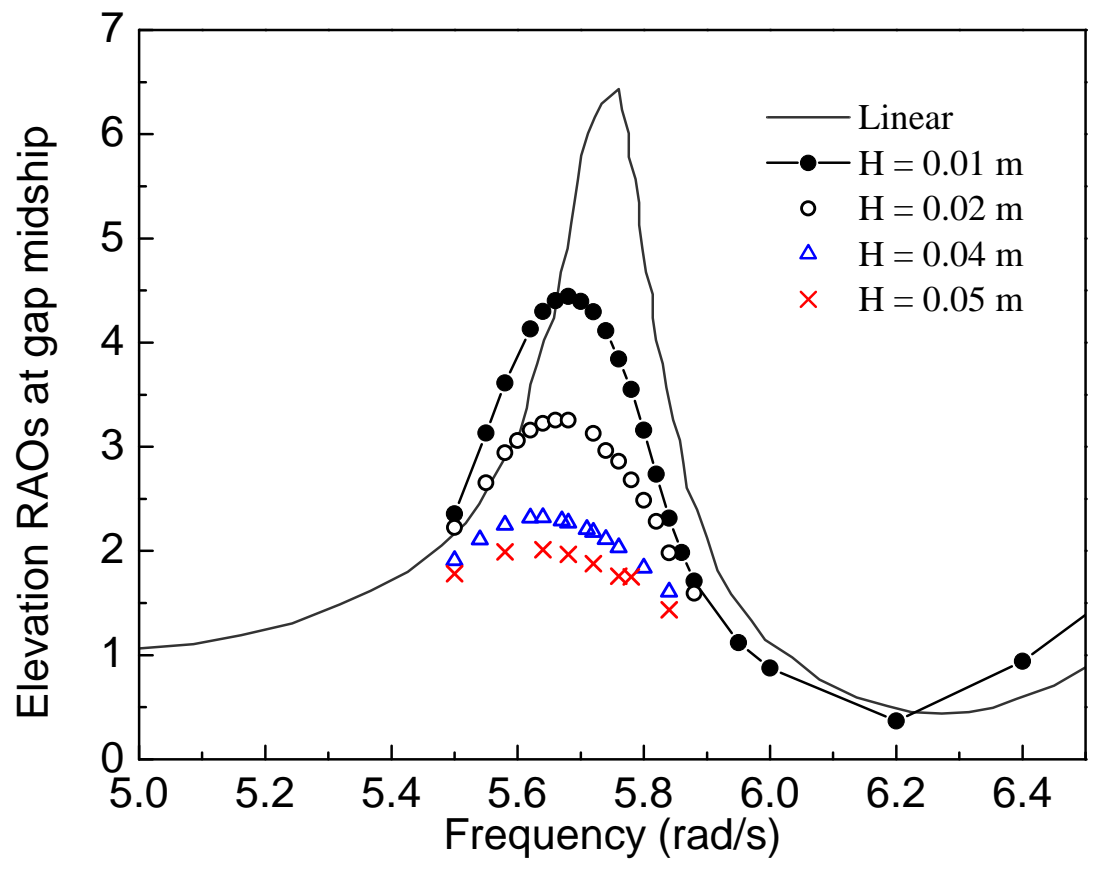

Figure 19: Effect of wave steepness on free surface RAOs at the mid-gap for barges of square bilges

resonant fluid will not be significantly influenced. To compare the velocity distribution near the gap inlet with the previous square bilge case in Fig. 15, we keep the incident wave frequency as $5.70 \mathrm{rad} / \mathrm{s}$ and wave hight $H=0.01 \mathrm{~m}$. Fig. 20 shows the velocity distribution at four moments: $t=38.4 \mathrm{~s}, 38.7 \mathrm{~s}, 38.9 \mathrm{~s}$ and 39.2 s. In contrast to the flow concentration at the gap inlet in Fig. 15(a), the flow pattern in Fig. 20(a) is rather smooth and no separation is observed. More obviously, no flow rotation or vortex appears when the gap elevation reaches its maximum in Fig. 20(b) whereas a pair of vortices almost fill up the gap inlet for the square bilges in Fig. 15(d) As there is no flow separation and vortex shedding around the rounded bilges, flow pattens of the 'out-pumped' fluid in Fig. 20(c)] are similar to the "in-pumped" fluid except the opposite velocity direction.

The vorticity iso-surface in Fig. 21 shows consistency with the velocity distribution that no vortex has been formed near the gap inlet. Most of the vortices are confined in a very thin boundary layer due to the viscous shear over the solid surface. Within the fluid bulk, viscous effect may be neglected. This suggests that the potential flow model may produce accurate estimations for the case of rounded bilges where the viscous damping induced around the bilges is negligible. Comparison of free surface RAOs at the mid-gap is presented in Fig. 22 and three incident wave amplitudes are included. The discrepancy in the peak RAO between the linear potential flow model and the present CFD model now is much reduced, compared with the square bilge barges in Fig. 19. Notably, similar to the square bilge case, the present CFD simulation again shows a broader frequency range for the piston mode resonance and the resonant frequency is slightly lower than the linear prediction. It has to be mentioned that the frequency resolution in the linear potential 


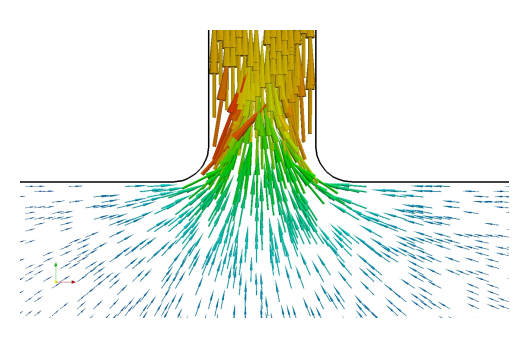

(a) $\mathrm{t}=38.4 \mathrm{~s}$

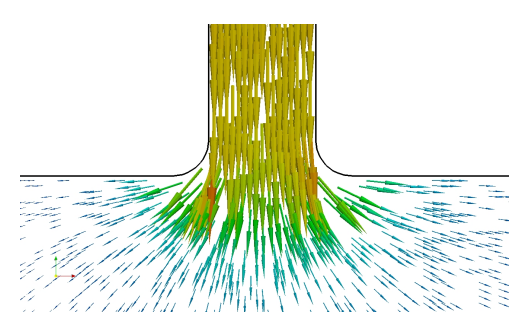

(c) $\mathrm{t}=38.9 \mathrm{~s}$

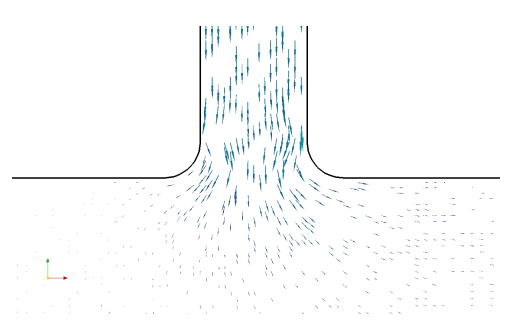

(b) $\mathrm{t}=38.7 \mathrm{~s}$

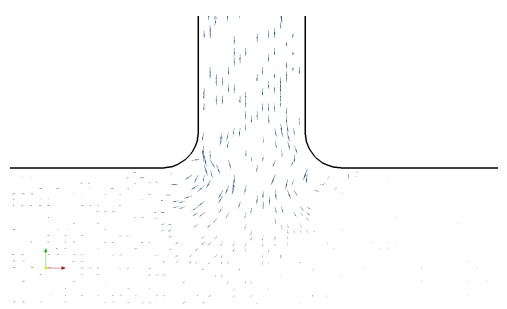

(d) $\mathrm{t}=39.2 \mathrm{~s}$

Velocity Magnitude $(\mathrm{m} / \mathrm{s})$

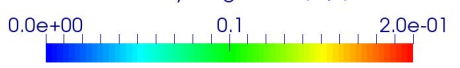

Figure 20: Velocity vectors around the barges of rounded bilge in the gap at different time instants

flow model is $0.05 \mathrm{rad} / \mathrm{s}$, such that any result within the frequency interval may be missing. To avoid that, in the present simulations we use a finer resolution $0.02 \mathrm{rad} / \mathrm{s}$ near the resonant frequency. Nonetheless, it becomes clear that flow separation accounts for the discrepancy in the peak RAO between potential models and measurements, and the statement on over-prediction using potential models only holds for the case of square bilge barges.
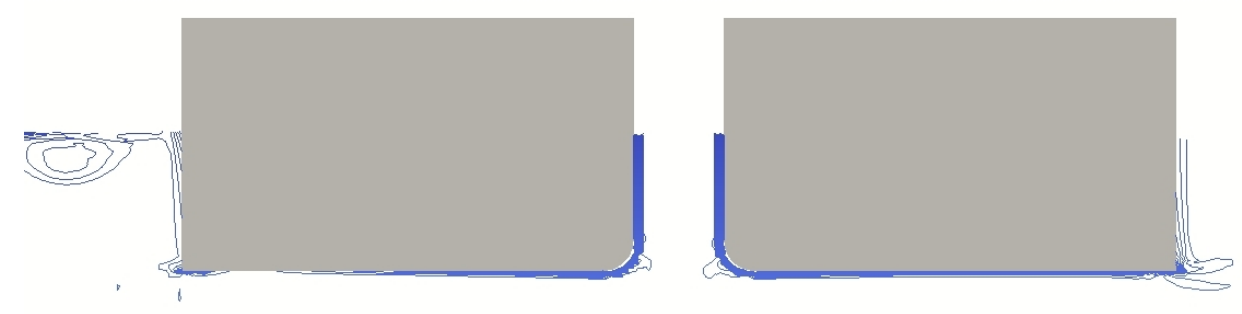

Figure 21: Vorticity iso-surface around the gap for barges of rounded bilge at the station $y=0$

It is shown in Fig. 22 that the surface RAOs for $H=0.01 \mathrm{~m}$ and $H=0.02 \mathrm{~m}$ are very close to each other, indicating a small difference in the system damping for the increased wave height. In contrast, a relatively large drop of peak RAO is observed for the case of square bilges. Increasing the wave height to $H$ $=0.04 \mathrm{~m}$, it seems the system damping also becomes relatively stronger, such that the peak RAO reduces 


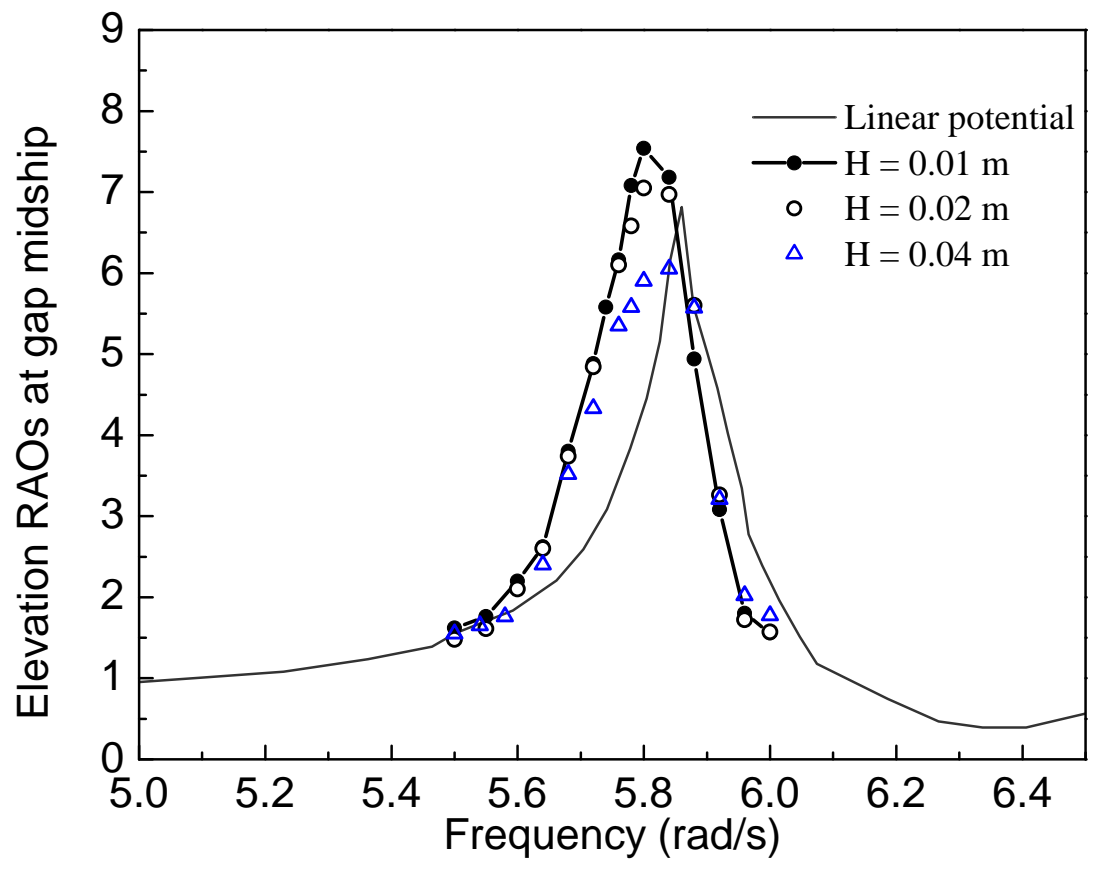

Figure 22: Free surface RAOs at the mid-gap for barges of rounded bilge

from 7.5 to 6.0. To have a clear picture of damping strength for different wave steepnesses, we plot the time histories of non-dimensional wave elevation at the mid-gap upon resonance for barges of both square bilge and rounded bilge in Fig. 23(a) and Fig. 23(b) respectively. The resonant frequency is about $5.70 \mathrm{rad} / \mathrm{s}$ for the square bilge and $5.80 \mathrm{rad} / \mathrm{s}$ for the rounded bilge. The responses near the resonance for $H=0.01 \mathrm{~m}$ and $0.02 \mathrm{~m}$ are slightly higher than the potential flow prediction, which is unexpected. As the viscous damping in the system reduces and the time needed to reach the steady state is almost doubled, it is possible that the reflection from the side walls becomes significant after a long-time simulation. Nonetheless, using much wider tank makes the present computation impractical.

The strength of system damping can be reflected from two aspects: one is the amplification $\eta_{s} / A$ and the other is the time it takes to reach its steady state $t_{\text {steady. }}$. Apparently the responses for rounded bilge barges are generally more amplified, for all wave heights, and the time needed to achieve the steady state is nearly doubled compared to square bilge barges. Further, the effect of wave steepness is more significant for square bilge barges. The details of wave steepness effect on the two cases are presented in Table. 3 and Table. 4 For the square bilge, increasing the incident wave height from $H=0.01 \mathrm{~m}$ to $H=0.02 \mathrm{~m}$ and $H=0.04 \mathrm{~m}$, it needs $23.3 \%$ and $40 \%$, respectively, lesser time to reach the steady state. The corresponding drops in the steady state response are $26.8 \%$ and $48.9 \%$. The reduction percentages of $t_{\text {steady }}$ and $\eta_{s} / A$ are lower when the bilge of the barges is rounded. It seems the reduction of both $t_{\text {steady }}$ and $\eta_{s} / A$ is correlated, and linked to the system damping strength that is a function of the incident wave steepness. Further exploration of quantitative assessment of the associated system damping is needed in the next stage of research. 
Table 3: Effect of wave steepness on the gap resonant response for barges of square bilge

\begin{tabular}{l|llll}
\hline Wave Heights & $t_{\text {steady }}$ & Time drop & $\eta_{s} / A$ & Response drop \\
\hline $\mathrm{H}=0.01 \mathrm{~m}$ & $30 \mathrm{~s}$ & - & 4.44 & - \\
$\mathrm{H}=0.02 \mathrm{~m}$ & $23 \mathrm{~s}$ & $23.3 \%$ & 3.25 & $26.8 \%$ \\
$\mathrm{H}=0.04 \mathrm{~m}$ & $18 \mathrm{~s}$ & $40 \%$ & 2.27 & $48.9 \%$ \\
\hline
\end{tabular}

Table 4: Effect of wave steepness on the gap resonant response for barges of rounded bilge

\begin{tabular}{l|llll}
\hline Wave Heights & $t_{\text {steady }}$ & Time drop & $\eta_{s} / A$ & Response drop \\
\hline $\mathrm{H}=0.01 \mathrm{~m}$ & $56 \mathrm{~s}$ & - & 7.54 & - \\
$\mathrm{H}=0.02 \mathrm{~m}$ & $51 \mathrm{~s}$ & $8.9 \%$ & 7.05 & $6.5 \%$ \\
$\mathrm{H}=0.04 \mathrm{~m}$ & $40 \mathrm{~s}$ & $28.6 \%$ & 5.90 & $21.8 \%$ \\
\hline
\end{tabular}

\section{Concluding remarks}

CFD simulations based on the OpenFOAM ${ }^{\circledR}$ platform in this study show promising results, especially for the prediction of wave resonance in a narrow gap between two side-by-side barges. The relaxation zone technique for wave generation and absorption is illustrated effective in a numerical wave tank. We firstly validate the numerical tank model in a $2 \mathrm{D}$ case of two boxes. Better agreement is achieved by the present viscous flow model at the gap resonance where the linear potential flow model generates unreasonable predictions. To explore the physical reason for the discrepancy between the linear model and the experiments, we present the detailed flow structure around the gap upon resonance. It is found that remarkable flow separation occurs around the corners of the boxes followed by vortex shedding and dissipation. This process in each wave period transfers a large amount of fluid kinetic energy into thermal energy, and it cannot be taken into account in any potential flow model.

To study a more realistic situation, 3D simulations are performed for two side-by-side fixed floating barges. Again we show detailed flow characteristics upon gap resonance at the piston mode. Two pairs of vortices, within and beneath the gap, are formed within the half period of water column oscillation. They contact, merge, and eventually dissipate through viscous shear. It is confirmed that the system viscous damping is induced by the sharp barge bilges; the flow separation accounts for most of the energy loss during the process of gap resonance. This further confirms the conclusion that the viscous effect plays a major role in explaining the discrepancy of resonant response between linear predictions and measurements.

As clear is the occurrence of flow separation at the sharp bilges, it is then of interest to investigate the same barges of rounded bilge. As expected, the linear potential flow model generates very close results with the CFD model for the peak response. No flow separation and vortex shedding are observed, indicating that the viscous effect can be neglected and the potential flow model remains valid for rounded bilge barges in the cases studied. 


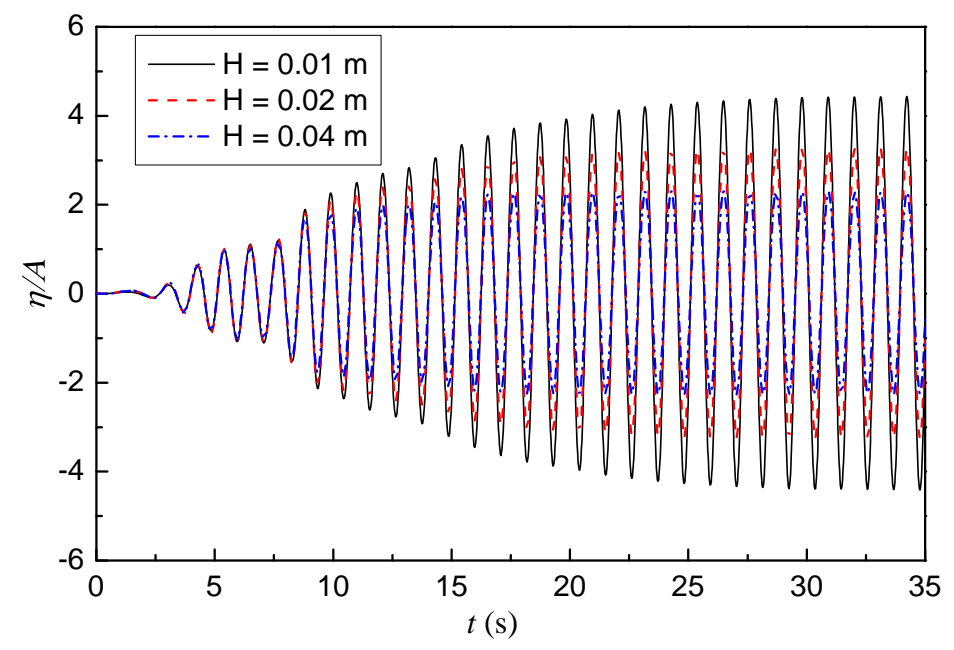

(a) Square bilges

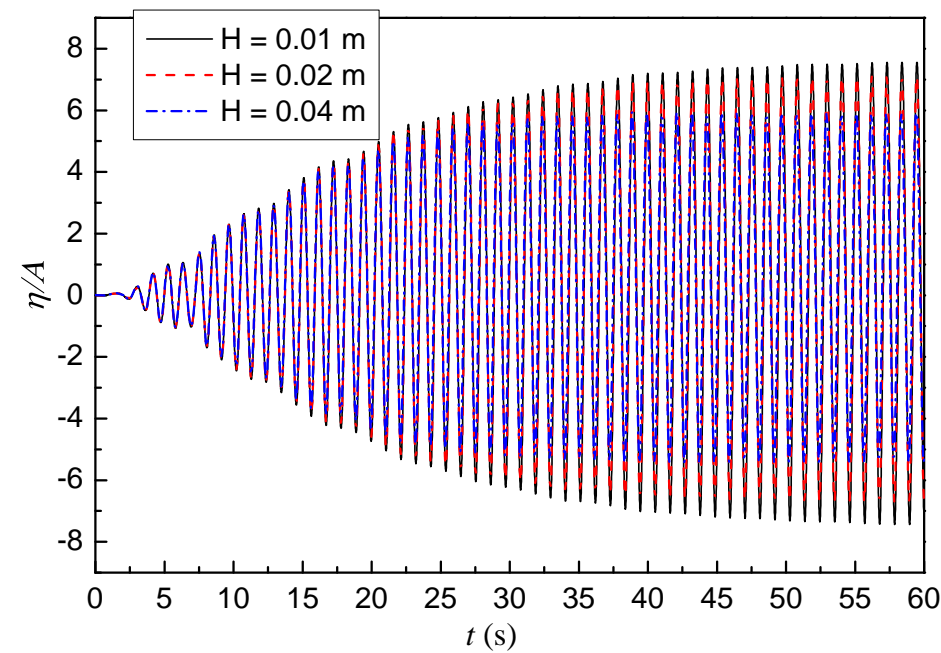

(b) Rounded bilges

Figure 23: Time histories of wave elevation in the gap at resonance for varying wave steepnesses

Lastly, the viscous damping associated with the twin-barge system is demonstrated dependent on the incident wave steepness. This dependence is stronger for the barges of square bilge and is weakened for that of rounded bilge. With increasing the wave steepness, both the time needed to reach the steady state and the resonant response are reduced. The reduction is more pronounced for the case of square bilge than the case of rounded bilge. 


\section{Acknowledgements}

The authors gratefully acknowledge the financial support provided by the Maritime and Port Authority of Singapore (MPA) Grant R-261-502-006-592.

\section{References}

Berberovic, E., van Hinsberg, N.P., Jakirlic, S., Roisman, I.V., Tropea, C., 2009. Drop impact onto a liquid layer of finite thickness: Dynamics of the cavity evolution. Physical Review E Statistical, Nonlinear, and Soft Matter Physics 79, 036306(1-15). doi/http://10.1103/PhysRevE.79.036306

Buchner, B., van Dijk, A., de Wilde, J., 2001. Numerical multiple-body simulations of side-by-side mooring to an fpso. Proceedings of the Eleventh International Offshore and Polar Engineering Conference 1, 343 353.

Chen, X.B., 2005. Hydrodynamic analysis for offshore lng terminals. Proceedings of 2nd International Workshop on Applied Offshore Hydrodynamics, Rio de Janeiro .

Chua, K.H., Eatock Taylor, R., Choo, Y.S., 2016. Hydrodynamics of side-by-side fixed floating bodies. Proceedings of the 35th International Conference on Ocean, Offshore and Arctic Engineering (OMAE) .

Faltinsen, O., Timokha, A., 2015. On damping of two-dimensional piston-mode sloshing in a rectangular moonpool under forced heave motions. Journal of Fluid Mechanics 772. doi $10.1017 / \mathrm{jfm} .2015 .234$.

Feng, X., Bai, W., 2015. Wave resonances in a narrow gap between two barges using fully nonlinear numerical simulation. Applied Ocean Research 50, 119 - 129. doi http://dx.doi.org/10.1016/j.apor.2015.01. 003 .

Fournier, J.R., Naciri, M., Chen, X.B., 2006. Hydrodynamics of two side-by-side vessels - experiments and numerical simulations. Proceedings of the Sixteenth International Offshore and Polar Engineering Conference 1, $158-165$.

Ganesan, S.T., Sen, D., 2016. Time domain simulation of side-by-side floating bodies using a 3d numerical wave tank approach. Applied Ocean Research 58, 189 - 217.

Huijsmans, R.H.M., Pinkster, J.A., de Wilde, J., 2001. Diffraction and radiation of waves around side-by-side moored vessels. Proceedings of the Eleventh International Offshore and Polar Engineering Conference 1, $406-412$.

Jacobsen, N.G., Fuhrman, D.R., Fredse, J., 2012. A wave generation toolbox for the open-source cfd library: Openfoam. International Journal for Numerical Methods in Fluids 70, 1073 - 1088. doi:http: //dx.doi.org/10.1002/fld.2726. 
Jasak, H., 1996. Error analysis and estimation in the finite volume method with applications to fluid flows. $\mathrm{PhD}$ thesis. Imperial College, London .

Kashiwagi, M., Endo, K., Yamaguchi, H., 2005. Wave drift forces and moments on two ships arranged side by side in waves. Ocean Engineering 32, 529 - 555. doi http://dx.doi.org/10.1016/j.oceaneng.2004. 09.005

Kristiansen, T., Faltinsen, O., 2008. Application of a vortex tracking method to the piston-like behaviour in a semi-entrained vertical gap. Applied Ocean Research 30,1 - 16. doi http://dx.doi.org/10.1016/j. apor.2008.02.003.

Kristiansen, T., Faltinsen, O.M., 2012. Gap resonance analyzed by a new domain-decomposition method combining potential and viscous flow draft. Applied Ocean Research 34, 198 - 208. doi:http://dx.doi. org/10.1016/j.apor.2011.07.001.

Lewandowski, E.M., 2008. Multi-vessel seakeeping computations with linear potential theory. Ocean Engineering 35, 1121 - 1131. doi http://dx.doi.org/10.1016/j.oceaneng.2008.04.011.

Li, Y., Zhang, C., 2016. Analysis of wave resonance in gap between two heaving barges. Ocean Engineering $117,210-220$.

Lu, L., Cheng, L., Teng, B., Li, Y., 2008. Numerical simulation of hydrodynamic resonance in a narrow gap between twin bodies subject to water waves. Proceedings of the Eighteenth International Offshore and Polar Engineering Conference, Vancouver, Canada .

Lu, L., Cheng, L., Teng, B., Sun, L., 2010. Numerical simulation and comparison of potential flow and viscous fluid models in near trapping of narrow gaps. Journal of Hydrodynamics, Ser. B 22, 120 - 125. doi:http://dx.doi.org/10.1016/S1001-6058(09)60180-3.

Molin, B., 2001. On the piston and sloshing modes in moonpools. Journal of Fluid Mechanics 430, $27-50$.

Molin, B., Remy, F., Camhi, A., Ledoux, A., 2009. Experimental and numerical study of the gap resonances in-between two rectangular barges. Proceedings of the 13th Congress of International Maritime Association of Mediterranean (IMAM) .

Moradi, N., Zhou, T., Cheng, L., 2016. Two-dimensional numerical study on the effect of water depth on resonance behaviour of the fluid trapped between two side-by-side bodies. Applied Ocean Research 58, $218-231$.

Newman, J.N., 2004. Progress in wave load computations on offshore structures. The 23rd International Conference on Offshore Mechanics and Arctic Engineering (Invited lecture), Vancouver, Canada .

Newman, J.N., Sclavounos, P.D., 1988. The computation of wave loads on large offshore structures. Proceedings of the International Confernce on Behaviour of Offshore Structures (BOSS88) 2, $605-619$. 
Pauw, W.H., Huijsmans, R.H.M., Voogt, A., 2007. Advances in the hydrodynamics of side-by-side moored vessels. Proceedings of the 26th International Conference on Offshore Mechanics and Arctic Engineering $4,597-603$.

Saitoh, T., Miao, G.P., Ishida, H., 2006. Theoretical analysis on appearance condition of fluid resonance in a narrow gap between two modules of very large floating structure. Proceedings of the Third Asia-Pacific Workshop on Marine Hydrodynamics, 170 - 175.

Sun, L., Eatock Taylor, R., Taylor, P.H., 2010. First- and second-order analysis of resonant waves between adjacent barges. Journal of Fluids and Structures 26, $954-978$. doi http://dx.doi.org/10.1016/j. jfluidstructs.2010.06.001.

Tan, L., Lu, L., Tang, G.Q., Cheng, L., Chen, X.B., 2017. An energy dissipation model for wave resonance problems in narrow gaps formed by floating structures. Proceedings of the 32nd International Workshop on Water Waves and Floating Bodies .

Teigen, P., Niedzwecki, J.M., 2006. A computational study of wave effects related to side-by-side lng offloading. Proceedings of the Sixteenth International Offshore and Polar Engineering Conference 2, 238 247.

Ubbink, O., Issa, R., 1999. A method for capturing sharp fluid interfaces on arbitrary meshes. Journal of Computational Physics 153, 26 - 50. doi:http://dx.doi.org/10.1006/jcph.1999.6276.

Watai, R., Dinoi, P., Ruggeri, F., Souto-Iglesias, A., Simos, A., 2015. Rankine time-domain method with application to side-by-side gap flow modeling. Applied Ocean Research 50,69-90. doi:http://dx.doi. org/10.1016/j.apor.2014.12.002.

Yeung, R.W., Seah, R.K.M., 2007. On helmholtz and higher-order resonance of twin floating bodies. Journal of Engineering Mathematics 58, 251 - 265. doi http://dx.doi.org/10.1007/s10665-006-9109-3.

Zhao, W., Wolgamot, H.A., Taylor, P.H., Eatock Taylor, R., 2017. Gap resonance and higher harmonics driven by focused transient wave groups. Journal of Fluid Mechanics 812, 905939. doi 10.1017/jfm. 2016.824

Zhu, H., Zhu, R., Miao, G., 2008. A time domain investigation on the hydrodynamic resonance phenomena of 3-d multiple floating structures. Journal of Hydrodynamics, Ser. B 20, 611 - 616. doi:http://dx.doi. org/10.1016/S1001-6058(08)60102-X 